\title{
Hyperbolicity of Generic Surfaces of High Degree in Projective 3-Space
}

\author{
Jean-Pierre Demailly and Jawher El Goul \\ Institut Fourier, Université de Grenoble I \\ 38402 Saint-Martin d'Hères, France
}

\section{Introduction}

The goal of this paper is to study the hyperbolicity of generic hypersurfaces in projective space. Recall that, by a well-known criterion due to Brody [Bro78], a compact complex space $X$ is hyperbolic in the sense of Kobayashi [Ko70] if and only if there is no nonconstant holomorphic map from $\mathbb{C}$ to $X$. More than twenty years ago, Shoshichi Kobayashi proposed the following famous conjecture: A generic $n$-dimensional hypersurface of large enough degree in $\mathbb{P}_{\mathbb{C}}^{n+1}$ is hyperbolic. This is of course obvious in the case of curves: the uniformization theorem shows that a smooth curve is hyperbolic if and only if it has genus at least 2 , which is the case if the degree is at least 4.

However, the picture is not at all clear in dimension $n \geq 2$. In view of results by Zaidenberg [Zai87], the most optimistic lower bound for the degree of hyperbolic $n$-dimensional hypersurfaces in $\mathbb{P}_{\mathbb{C}}^{n+1}$ would be $2 n+1$ (assuming $n \geq 2$ ). The hyperbolicity of $X$ in Kobayashi's analytic setting is expected to be equivalent to the purely algebraic fact that $X$ does not contain any subvariety not of general type (it does imply e.g. that $X$ has no rational curve and no nontrivial image of abelian varieties). L. Ein has shown in [Ein87] that a very generic hypersurface of $\mathbb{P}_{\mathbb{C}}^{n+1}$ of degree at least $2 n+2$ does not contain any submanifold not of general type; a simpler proof has been given later by C. Voisin [Voi96]. The above algebraic property looks however substantially weaker than Kobayashi hyperbolicity because it only constrains the geometry of algebraic subvarieties rather than that of general entire transcendental maps.

In the case of a surface $X$, the optimal degree lower bound for hyperbolicity is expected to be equal to 5 , which is also precisely the lowest possible degree for $X$ to be of general type. In fact, Green-Griffiths [GG80] have formulated the following much stronger conjecture: If $X$ is a variety of general type, every entire curve $f: \mathbb{C} \rightarrow X$ is algebraically degenerate, and (optimistic version of the conjecture) there is a proper algebraic subset $Y \subset X$ containing all images of nonconstant entire curves. As a (very) generic surface of degree at least 5 does not contain rational or elliptic curves by the results of $\mathrm{H}$. Clemens ([Cl86], [CKM88]) and G. Xu [Xu94], it would then follow that such a surface is hyperbolic. However, almost nothing was known before for the case of transcendental curves 
drawn on a (very) generic surface or hypersurface. Only rather special examples of hyperbolic hypersurfaces have been constructed in higher dimensions, thanks to a couple of techniques due to Brody-Green [BG78], Nadel [Na89], Masuda-Noguchi [MN94], Demailly-El Goul [DEG97] and Siu-Yeung [SY97]. The related question of complements of curves in $\mathbb{P}^{2}$ has perhaps been more extensively investigated, see Zaidenberg [Zai89, 93], Dethloff-Schumacher-Wong [DSW92, 94], Siu-Yeung [SY95], Dethloff-Zaidenberg [DZ95a,b].

Here, we will obtain a confirmation of Kobayashi's conjecture in dimension 2 , for the case of surfaces of degree at least 20. Our analysis is based on more general results, which also apply to surfaces not necessarily embedded in $\mathbb{P}^{3}$. Before presenting them, we introduce some useful terminology. Let

$$
f:(\mathbb{C}, 0) \rightarrow X
$$

be a germ of curve on a surface $X$, expressed as $f=\left(f_{1}, f_{2}\right)$ in suitable local coordinates. The notation $E_{k, m} T_{X}^{\star}$ stands for the sheaf of "invariant" jet differentials of order $k$ and total degree $m$, which will be defined in greater detail in $\S 1$. For the sake of simplicity, we describe here the simpler case of jet differentials of order 2. A section of $E_{2, m} T_{X}^{\star}$ is a polynomial differential operator of the form

$$
P(f)=\sum_{\alpha_{1}+\alpha_{2}+3 j=m} a_{\alpha_{1} \alpha_{2} j}(f) f_{1}^{\prime \alpha_{1}} f_{2}^{\prime \alpha_{2}}\left(f_{1}^{\prime} f_{2}^{\prime \prime}-f_{1}^{\prime \prime} f_{2}^{\prime}\right)^{j}
$$

acting on germs of curves. It is clear that $\bigoplus E_{2, m} T_{X}^{\star}$ is a graded algebra. An algebraic multi-foliation on a surface $X$ is by definition associated with a rank 1 subsheaf $\mathcal{F} \subset S^{m} T_{X}^{\star}$. Such a subsheaf $\mathcal{F}$ is generated locally by a jet differential of order 1, i.e. a section $s \in \Gamma\left(U, S^{m} T_{X}^{\star}\right)$ of the form

$$
s(z)=\sum_{0 \leq j \leq m} a_{j}\left(z_{1}, z_{2}\right)\left(d z_{1}\right)^{m-j}\left(d z_{2}\right)^{j},
$$

vanishing at only finitely many points, and such that

$$
s(z)=\prod_{1 \leq j \leq m}\left(c_{1, j}(z) d z_{1}+c_{2, j}(z) d z_{2}\right)
$$

factorizes as a product of generically distinct linear forms. Equivalently, the foliation is defined by a collection of $m$-lines in $T_{X, z}$ at each generic point $z$, so that it is associated with a (possibly singular) surface $Y \subset P\left(T_{X}\right)$ which is $m$-sheeted over $X$. Of course, if $\widetilde{Y}$ is a desingularization of $Y$, then $\widetilde{Y}$ carries an associated (possibly singular) foliation, that is, a rank 1 subsheaf of $T_{\widetilde{Y}}^{\star}$. A leaf of the multi-foliation on $X$ is just the projection to $X$ of a leaf of the corresponding foliation on $\widetilde{Y}$. We further introduce the following definition.

Definition. - Let $X$ be a nonsingular projective variety of general type. We define the $k$-jet threshold $\theta_{k}$ of $X$ to be the infimum

$$
\theta_{k}=\inf _{m>0} \theta_{k, m} \in \mathbb{R},
$$

where $\theta_{k, m}$ is the smallest rational number $t / m$ such that there is a non zero section in $H^{0}\left(X, E_{k, m} T_{X}^{\star} \otimes \mathcal{O}\left(t K_{X}\right)\right.$ ) (assuming that $t K_{X}$ is an integral divisor, $t \in \mathbb{Q})$. 
Since $E_{k, m} T_{X}^{\star} \subset E_{k+1, m} T_{X}^{\star}$, we have of course

$$
\theta_{1} \geq \theta_{2} \geq \ldots \geq \theta_{k} \geq \ldots
$$

If $\theta_{1}<0$, the variety $X$ possesses a lot of 1 -jet differentials, i.e. sections of $H^{0}\left(X, S^{m} T_{X}^{\star}\right)$, and the theory becomes much easier. The core of the present paper is to investigate the situation $\theta_{1} \geq 0, \theta_{2}<0$. It turns out that nonsingular surfaces of $\mathbb{P}^{3}$ enter in this category when the degree is at least 15 . Degrees in the range $[5,14]$ would (a priori) only yield $\theta_{k}<0$ for values of $k$ at least equal to 3 , and the situation becomes harder to study as $k$ increases.

Main Theorem. - Let $X$ be a nonsingular surface of general type, and let $\theta_{k}$ be its $k$-jet threshold, $k \geq 1$. Assume that either $\theta_{1}<0$ or that the following three conditions are satisfied:

(a) $\theta_{1} \geq 0, \theta_{2}<0$;

(b) $\operatorname{Pic}(X)=\mathbb{Z}$;

(c) The Chern numbers of $X$ satisfy $\frac{c_{1}^{2}}{c_{2}}>\frac{9}{13+12 \theta_{2}}$.

Then every nonconstant holomorphic map $f: \mathbb{C} \rightarrow X$ is a leaf of an algebraic multi-foliation on $X$.

Our strategy is based on a careful analysis of the geometry of Semple jet bundles, as proposed in [Dem95]. Following an idea suggested by Green-Griffiths [GG80], we use Riemann-Roch calculations to prove the existence of suitable 2jet differentials of sufficiently large degree. Actually, it can be shown that the condition $\theta_{2}<0$ always holds true under the assumption $13 c_{1}^{2}-9 c_{2}>0$. Now, any 2 -jet differential equation corresponds to a divisor $Z$ in the (4-dimensional) Semple 2-jet bundle $X_{2}$. We apply Riemann-Roch again on that divisor $Z$ to show that the base locus of 2-jets is at most 2-dimensional - this is exactly the place where condition (c) is needed. From this, the existence of the asserted algebraic multi-foliation follows.

In order to apply the Main Theorem, we still have to check that conditions (a), (b), (c) are met for a (very) generic surface in $\mathbb{P}^{3}$ of sufficiently high degree. Here, the terminology "generic" (resp. "very generic") is used to indicate that the exceptional set is contained in a finite (resp. countable) union of algebraic subsets in the moduli space of surfaces in $\mathbb{P}^{3}$. We prove the following results (see sections 3 to 6$)$.

Proposition 1. - Let $X$ be a nonsingular surface of general type such that $\operatorname{Pic}(X)=\mathbb{Z}$ and $\theta_{1} \geq 0$. Then

$$
\theta_{2} \geq \min \left(\theta_{2,3}, \theta_{2,4}, \theta_{2,5}, \frac{1}{2} \theta_{1}-\frac{1}{6}\right) \text {. }
$$

Proposition 2. - Let $X$ be a nonsingular surface of degree $d \geq 5$ in $\mathbb{P}^{3}$. Then

(a) $c_{1}^{2}=d(d-4)^{2}, \quad c_{2}=d\left(d^{2}-4 d+6\right)$. 
(b) $\operatorname{Pic}(X)=\mathbb{Z}$ if $X$ is very generic (Noether-Lefschetz theorem).

(c) $\frac{1}{d-4} \leq \theta_{1} \leq \frac{2}{d-4}$.

(d) $\theta_{2}<0$ for $d \geq 15$.

(e) For a generic surface of degree $d \geq 6, \theta_{2, m} \geq-\frac{1}{2 m}+\frac{2-7 / 2 m}{d-4}$ if $m=3,4,5$.

(f) For a very generic surface of degree $d \geq 6, \theta_{2} \geq-\frac{1}{6}+\frac{1}{2(d-4)}$.

(g) Condition (c) of the Main Theorem is met for a very generic surface of degree $d \geq 21$.

Property (c) of Proposition 2 is verified through an explicit calculation of sections, made in $\S 5$. Property $(\mathrm{d})$ is a consequence of the fact that $13 c_{1}-9 c_{2}>0$ for $d \geq 15$. In order to check property (e), we rely on an elementary but very useful "proportionality lemma". We are indebted to Mihai Paun for a substantial improvement of the earlier statement of our proportionality lemma. Let us first observe that there is a natural filtration on $E_{2, m} T_{X}^{\star}$, defined by the degree $j$ of the monomials $\left(f_{1}^{\prime} f_{2}^{\prime \prime}-f_{1}^{\prime \prime} f_{2}^{\prime}\right)^{j}$ in $P$, inducing an exact sequence

$$
0 \longrightarrow S^{m} T_{X}^{\star} \longrightarrow E_{2, m} T_{X}^{\star} \stackrel{\Phi}{\longrightarrow} E_{2, m-3} T_{X}^{\star} \otimes K_{X} \rightarrow 0
$$

Proportionality lemma. - Let $X$ be a nonsingular surface of general type. Then, for all sections

$$
P_{i} \in H^{0}\left(X, E_{2, m_{i}} T_{X}^{\star} \otimes \mathcal{O}_{X}\left(t_{i} K_{X}\right)\right)
$$

with $m_{i}=3,4,5$ and $t_{i} \in \mathbb{Q}_{+}, 1+t_{1}+t_{2}<\left(m_{1}+m_{2}-3\right) \theta_{1, m_{1}+m_{2}-3}$, the section

$$
\beta_{1} P_{2}-\beta_{2} P_{1} \in H^{0}\left(X, E_{2, m_{1}+m_{2}-3} T_{X}^{\star} \otimes \mathcal{O}_{X}\left(\left(1+t_{1}+t_{2}\right) K_{X}\right)\right)
$$

associated with $\beta_{i}=\Phi\left(P_{i}\right)$ vanishes.

The proportionality lemma has the very interesting feature that it can convert a nonvanishing theorem into a generic vanishing theorem! Actually, if one can produce examples of sections $P_{1}$ for $t_{1}$ sufficiently small, then there cannot exist sections $P_{2}$ for values of $t_{2}$ which are still smaller. The construction of meromorphic connections introduced by Nadel [Na89], as it turns out, does produce adequate sections $P_{1}$ with values in $\left.E_{2,3} T^{\star} \otimes \mathcal{O}\left(t_{1} K_{X}\right), t_{1} \in\right]-1,0[$, for certain very particular surfaces.

According to recent results of M. McQuillan (see section 6), the Main Theorem solves Kobayashi's conjecture in the case of surfaces.

Corollary 1. - A very generic surface $X$ in $\mathbb{P}^{3}$ of degree $d \geq 21$ is Kobayashi hyperbolic, that is, there is no nonconstant holomorphic map from $\mathbb{C}$ to $X$.

As a consequence of the proof, we also get

Corollary 2. - The complement of a very generic curve in $\mathbb{P}^{2}$ is hyperbolic and hyperbolically imbedded for all degrees $d \geq 21$. 
Our hope is that a suitable generalization of the present techniques to higher order jets will soon lead to a solution of the Green-Griffiths conjecture: every holomorphic map from $\mathbb{C}$ to a surface of general type is algebraically degenerate. We would like to thank Gerd Dethloff and Steven Lu for sharing generously their views on these questions, and Bernie Shiffman for interesting discussions on related subjects.

\section{Semple jet bundles}

Let $X$ be a complex $n$-dimensional manifold. According to Green-Griffiths [GG80], we let $J_{k} \rightarrow X$ be the bundle of $k$-jets of germs of parametrized curves in $X$, that is, the set of equivalence classes of holomorphic maps $f:(\mathbb{C}, 0) \rightarrow(X, x)$, with the equivalence relation $f \sim g$ if and only if all derivatives $f^{(j)}(0)=g^{(j)}(0)$ coincide for $0 \leq j \leq k$, when computed in some local coordinate system of $X$ near $x$. The projection map $J_{k} \rightarrow X$ is simply $f \mapsto f(0)$. Thanks to Taylor's formula, the fiber $J_{k, x}$ can be identified with the set of $k$-tuples of vectors $\left(f^{\prime}(0), \ldots, f^{(k)}(0)\right) \in\left(\mathbb{C}^{n}\right)^{k}$. It follows that $J_{k}$ is a holomorphic fiber bundle with typical fiber $\left(\mathbb{C}^{n}\right)^{k}$ over $X$ (however, $J_{k}$ is not a vector bundle for $k \geq 2$, because of the nonlinearity of coordinate changes). In the terminology of [Dem95], a directed manifold is a pair $(X, V)$ where $X$ is a complex manifold and $V \subset T_{X}$ a subbundle. Let $(X, V)$ be a complex directed manifold. We define $J_{k} V \rightarrow X$ to be the bundle of $k$-jets of germs of curves $f:(\mathbb{C}, 0) \rightarrow X$ which are tangent to $V$, i.e., such that $f^{\prime}(t) \in V_{f(t)}$ for all $t$ in a neighborhood of 0 , together with the projection map $f \mapsto f(0)$ onto $X$. It is easy to check that $J_{k} V$ is actually a subbundle of $J_{k}$. One of the essential tools used here are the projectivized jet bundles $X_{k} \rightarrow X$ introduced in [Dem95]. Let $\mathbb{G}_{k}$ be the group of germs of $k$-jets biholomorphisms of $(\mathbb{C}, 0)$, that is, the group of germs of biholomorphic maps

$$
t \mapsto \varphi(t)=a_{1} t+a_{2} t^{2}+\cdots+a_{k} t^{k}, \quad a_{1} \in \mathbb{C}^{\star}, a_{j} \in \mathbb{C}, j \geq 2,
$$

in which the composition law is taken modulo terms $t^{j}$ of degree $j>k$. The group $\mathbb{G}_{k}$ acts on the left on $J_{k} V$ by reparametrization, $(\varphi, f) \mapsto f \circ \varphi$. The bundle $X_{k}$ can then be seen as a natural compactification of the quotient of the open subset of regular jets $J_{k} V^{\text {reg }} \subset J_{k} V$ by the action of $\mathbb{G}_{k}$. We recall here briefly the basic construction.

To a directed manifold $(X, V)$, one associates inductively a sequence of directed manifolds $\left(X_{k}, V_{k}\right)$ as follows. Starting with $\left(X_{0}, V_{0}\right)=(X, V)$, one sets inductively $X_{k}=P\left(V_{k-1}\right)[P(V)$ stands for the projectivized bundle of lines in the vector bundle $V]$, where $V_{k}$ is the subbundle of $T_{X_{k}}$ defined at any point $(x,[v]) \in X_{k}, v \in V_{k-1, x}$, by

$$
V_{k,(x,[v])}=\left\{\xi \in T_{X_{k},(x,[v])} ;\left(\pi_{k}\right)_{\star} \xi \in \mathbb{C} \cdot v\right\}, \quad \mathbb{C} \cdot v \subset V_{k-1, x} \subset T_{X_{k-1}, x} .
$$

Here $\pi_{k}: X_{k} \rightarrow X_{k-1}$ denotes the natural projection. We denote by $\mathcal{O}_{X_{k}}(-1)$ the tautological line subbundle of $\pi_{k}^{\star} V_{k-1}$, such that

$$
\mathcal{O}_{X_{k}}(-1)_{(x,[v])}=\mathbb{C} \cdot v,
$$


for all $(x,[v]) \in X_{k}=P\left(V_{k-1}\right)$. By definition, the bundle $V_{k}$ fits in an exact sequence

$$
0 \longrightarrow T_{X_{k} / X_{k-1}} \longrightarrow V_{k} \stackrel{\pi_{k \star}}{\longrightarrow} \mathcal{O}_{X_{k}}(-1) \longrightarrow 0
$$

and the Euler exact sequence of $T_{X_{k} / X_{k-1}}$ yields

$$
0 \longrightarrow \mathcal{O}_{X_{k}} \longrightarrow \pi_{k}^{\star} V_{k-1} \otimes \mathcal{O}_{X_{k}}(1) \longrightarrow T_{X_{k} / X_{k-1}} \longrightarrow 0 .
$$

From these sequences, we infer

$$
\operatorname{rank} V_{k}=\operatorname{rank} V_{k-1}=\cdots=\operatorname{rank} V=r, \quad \operatorname{dim} X_{k}=n+k(r-1) .
$$

We say that $\left(X_{k}, V_{k}\right)$ is the $k$-jet directed manifold associated with $(X, V)$, and we let

$$
\pi_{k, j}=\pi_{j+1} \circ \cdots \circ \pi_{k-1} \circ \pi_{k}: X_{k} \longrightarrow X_{j}
$$

be the natural projection.

Now, let $f: \Delta_{r} \rightarrow X$ be a nonconstant tangent trajectory to $V$. Then $f$ lifts to a well defined and unique trajectory $f_{[k]}: \Delta_{r} \rightarrow X_{k}$ of $X_{k}$ tangent to $V_{k}$. Moreover, the derivative $f_{[k-1]}^{\prime}$ gives rise to a section

$$
f_{[k-1]}^{\prime}: T_{\Delta_{r}} \rightarrow f_{[k]}^{\star} \mathcal{O}_{X_{k}}(-1) .
$$

With any section $\sigma$ of $\mathcal{O}_{X_{k}}(m), m \geq 0$, on any open set $\pi_{k, 0}^{-1}(U), U \subset X$, we can associate a holomorphic differential operator $Q$ of order $k$ acting on $k$-jets of germs of curves $f:(\mathbb{C}, 0) \rightarrow U$ tangent to $V$, by putting

$$
Q(f)(t)=\sigma\left(f_{[k]}(t)\right) \cdot f_{[k-1]}^{\prime}(t)^{\otimes m} \in \mathbb{C} .
$$

In order to understand better this correspondence, let us use locally a coordinate chart and the associated trivialization $T_{X} \simeq \mathbb{C}^{n}$, so that the projection $\mathbb{C}^{n} \rightarrow \mathbb{C}^{r}$ onto the first $r$-coordinates gives rise to admissible coordinates on $V$. Then $f^{\prime}, f^{\prime \prime}, \ldots, f^{(k)}$ are in one to one correspondence with the $r$-tuples

$$
\left(f_{1}^{\prime}, \ldots, f_{r}^{\prime}\right), \quad\left(f_{1}^{\prime \prime}, \ldots, f_{r}^{\prime \prime}\right), \ldots\left(f_{1}^{(k)}, \ldots, f_{r}^{(k)}\right) .
$$

1.1. Proposition ([Dem95]). - The direct image sheaf $\left(\pi_{k, 0}\right)_{\star} \mathcal{O}_{X_{k}}(m)$ on $X$ coincides with the (locally free) sheaf $E_{k, m} V^{\star}$ of $k$-jet differentials of weighted degree $m$, that is, by definition, the set of germs of polynomial differential operators

$$
Q(f)=\sum_{\alpha_{1} \ldots \alpha_{k} \in \mathbb{N}^{r}} a_{\alpha_{1} \ldots \alpha_{k}}(f)\left(f^{\prime}\right)^{\alpha_{1}}\left(f^{\prime \prime}\right)^{\alpha_{2}} \cdots\left(f^{(k)}\right)^{\alpha_{k}}
$$

on $J_{k} V$ [in multi-index notation, $\left.\left(f^{\prime}\right)^{\alpha_{1}}=\left(f_{1}^{\prime}\right)^{\alpha_{1,1}}\left(f_{2}^{\prime}\right)^{\alpha_{1,2}} \ldots\left(f_{r}^{\prime}\right)^{\alpha_{1, r}}\right]$, which are moreover invariant under arbitrary changes of parametrization: a germ of operator $Q \in E_{k, m} V^{\star}$ is characterized by the condition that, for every germ $f \in J_{k} V$ and every germ $\varphi \in \mathbb{G}_{k}$,

$$
Q(f \circ \varphi)=\varphi^{\prime m} Q(f) \circ \varphi
$$

Observe that the weighted degree $m$ is taken with respect to weights 1 for $f^{\prime}$, 2 for $f^{\prime \prime}$, etc., thus counts the total numbers of "primes" in each monomial of the expansion of $Q$. 
A basic result, relying on the Ahlfors-Schwarz lemma, is that any entire curve $f: \mathbb{C} \rightarrow X$ tangent to $V$ must automatically satisfy all algebraic differential equations $Q(f)=0$ arising from global jet differential operators

$$
Q \in H^{0}\left(X, E_{k, m} V^{\star} \otimes \mathcal{O}(-A)\right)
$$

which vanish on some ample divisor $A$. More precisely, we have the following.

1.2. Theorem ([GG80], [Dem95], [SY97]). - Assume that there exist integers $k, m>0$ and an ample line bundle $A$ on $X$ such that

$$
H^{0}\left(X_{k}, \mathcal{O}_{X_{k}}(m) \otimes\left(\pi_{k, 0}\right)^{\star} A^{-1}\right) \simeq H^{0}\left(X, E_{k, m} V^{\star} \otimes A^{-1}\right)
$$

has nonzero sections $\sigma_{1}, \ldots, \sigma_{N}$. Let $Z \subset X_{k}$ be the base locus of these sections. Then every entire curve $f: \mathbb{C} \rightarrow X$ tangent to $V$ is such that $f_{[k]}(\mathbb{C}) \subset Z$. In other words, for every global $\mathbb{G}_{k}$-invariant polynomial differential operator $Q$ with values in $A^{-1}$, every entire curve $f$ tangent to $V$ must satisfy the algebraic differential equation $Q(f)=0$.

By definition, a line bundle $L$ is big if there exists an ample divisor $A$ on $X$ such that $L^{\otimes m} \otimes \mathcal{O}(-A)$ admits a nontrivial global section when $m$ is large (then there are lots of sections, namely $h^{0}\left(X, L^{\otimes m} \otimes \mathcal{O}(-A)\right) \gg m^{n}$ with $\left.n=\operatorname{dim} X\right)$.

As a consequence, Theorem 1.2 can be applied when $\mathcal{O}_{X_{k}}(1)$ is big. In the sequel, we will be concerned only with the "standard case" $V=T_{X}$.

A conjecture by Green-Griffiths and Lang states that every entire curve drawn on a variety of general type is algebraically degenerate, i.e. contained in a proper algebraic subvariety. In view of this conjecture and of Theorem 1.2, it is especially interesting to compute the base locus of the global sections of jet differentials, sometimes referred to in the litterature as the Green-Griffiths locus of $X$. According to the definition of invariant $k$-jets given in [Dem95], we introduce instead the base locus $B_{k}$ of invariant $k$-jets, that is, the intersection

$$
B_{k}:=\bigcap_{m>0} B_{k, m} \subset X_{k}
$$

of the base loci $B_{k, m}$ of all line bundles $\mathcal{O}_{X_{k}}(m) \otimes \pi_{k, 0}^{\star} \mathcal{O}(-A)$, where $A$ is a given arbitrary ample divisor over $X$ (clearly, $B_{k}$ does not depend on the choice of $A$ ). Our hope is that

$$
Y:=\bigcap_{k>0} \pi_{k, 0}\left(B_{k}\right) \subset X
$$

can always be shown to be a proper subvariety of $X$. In the present situation, this will be achieved by lowering down the dimension of $B_{k}$ as much as possible. For a surface, we will actually show that non vertical components of $B_{2}$ have dimension at least 2 under reasonable geometric assumptions on $X$. In general, our expectation is that non vertical components of $B_{k}$ have dimension at most equal to $\operatorname{dim} X$, whenever $X$ is of general type and $k$ is large enough. 


\section{Base locus of 1-jets}

From now on, we suppose that $X$ is a nonsingular surface of general type (in particular, $X$ must be algebraic, see [BPV84]), and let $c_{1}$ and $c_{2}$ be the Chern classes of $X$. We first describe some known facts about surfaces of general type with $c_{1}^{2}>c_{2}$, in connection with the existence of "symmetric differentials", i.e., sections in $E_{1, m} T_{X}^{\star}=S^{m} T_{X}^{\star}$. Section 3 will be devoted to refinements of these results in the case of order 2 jets.

The starting point is Hirzebruch's Riemann-Roch formula [Hi66]

$$
\chi\left(X, S^{m} T_{X}^{\star}\right)=\frac{m^{3}}{6}\left(c_{1}^{2}-c_{2}\right)+O\left(m^{2}\right) .
$$

On the other hand, Serre duality implies

$$
h^{2}\left(X, S^{m} T_{X}^{\star}\right)=h^{0}\left(X, S^{m} T_{X} \otimes K_{X}\right) .
$$

A vanishing theorem due to Bogomolov [Bo79] (see also e.g. [Dem95], § 14) implies that, on a surface $X$ of general type,

$$
h^{0}\left(X, S^{p} T_{X} \otimes K_{X}^{\otimes q}\right)=0 \quad \text { for all } p, q \text { such that } p-2 q>0 .
$$

In particular, $h^{0}\left(X, S^{m} T_{X} \otimes K_{X}\right)=0$ whenever $m \geq 3$ and we get

$$
h^{0}\left(P\left(T_{X}\right), \mathcal{O}_{P\left(T_{X}\right)}(m)\right)=h^{0}\left(X, S^{m} T_{X}^{\star}\right) \geq \chi\left(X, S^{m} T_{X}^{\star}\right) \geq \frac{m^{3}}{6}\left(c_{1}^{2}-c_{2}\right)+O\left(m^{2}\right) .
$$

As a consequence, the line bundle $\mathcal{O}_{X_{1}}(1)$ is big when $c_{1}^{2}>c_{2}$, and the base locus

$$
B_{1}=\bigcap_{m>0} \operatorname{Bs}\left|\mathcal{O}_{X_{1}}(m) \otimes \mathcal{O}(-A)\right|
$$

(which is equal in this case to the Green-Griffiths locus) is a proper algebraic subset of $X_{1}=P\left(T_{X}\right)$.

Let $Z$ be an irreducible component of $B_{1}$ which is a horizontal surface, i.e. such that $\pi_{1,0}(Z)=X$. Then the subbundle $V_{1} \subset T_{X_{1}}$ defines on the desingularization $\widetilde{Z}$ of $Z$ an algebraic foliation by curves, such that the tangent bundle to the leaves is given by $T_{Z} \cap V_{1}$ at a general point. Indeed, at any regular point $x_{1}=[v] \in Z$, $v \in T_{X, x}$, at which $\pi_{1,0}$ is a local biholomorphism onto $X, V_{1, x_{1}}$ consists of those vectors in $T_{X_{1}}$ which project to the line $\mathbb{C} v \subset T_{X, x}$, and $T_{Z, x_{1}} \cap V_{1}$ is the lifting of that line by the isomorphism $\left(\pi_{1,0}\right)_{\star}: T_{Z, x_{1}} \rightarrow T_{X, x}$.

By Theorem 1.2, for any nonconstant entire curve $f: \mathbb{C} \rightarrow X$, the curve $f_{[1]}$ must lie in some component $Z$ of $B_{1}$. If $Z$ is not horizontal, i.e. if $C=\pi_{1,0}(Z)$ is a curve in $X$, then $f(\mathbb{C}) \subset C$. Otherwise, we know by the above that $Z$ carries a canonical algebraic foliation, and that the image of $f_{[1]}$ lies either in the singular set of $Z$ or of the projection $\pi_{1,0}: Z \rightarrow X$ (which both consist of at most finitely many curves), or is a leaf of the foliation. Combining these observations with a theorem of A. Seidenberg [Se68] on desingularization of analytic foliations on surfaces, F. Bogomolov [Bo77] obtained the following finiteness theorem.

2.1. Theorem (Bogomolov). - There are only finitely many rational and elliptic curves on a surface of general type with $c_{1}^{2}>c_{2}$. 
Theorem 2.1 can now be seen (see [M-De78]) as a direct consequence of the following theorem of J.-P. Jouanolou [Jo78] on algebraic foliations, and of the fact that a surface of general type cannot be ruled or elliptic.

2.2. Theorem (Jouanolou). - Let $L$ be a subsheaf of the cotangent bundle of a projective manifold defining an analytic foliation of codimension 1. Let $H$ be the dual distribution of hyperplanes in $T_{X}$. If there is an infinite number of hypersurfaces tangent to $H$, then $H$ must be the relative tangent sheaf to a meromorphic fibration of $X$ onto a curve.

The above result of Bogomolov does not give information on transcendental curves. As observed by Lu and Yau [LY90], one can say more if the topological index $\frac{c_{1}^{2}-2 c_{2}}{3}$ is positive, using the following result of Y. Miyaoka [Mi82] on the almost everywhere ampleness of $T_{X}^{\star}$. We recall here their proof in order to point out the analogy with results of section 3 (see [ScTa85] for the general case of semi-stable vector bundles).

First recall that a line bundle $L$ on a projective manifold is called numerically effective (nef) if the intersection $L \cdot C$ is nonnegative for all curve $C$ in $X$. A surface $X$ of general type is called minimal if its canonical bundle $K_{X}$ is nef.

2.3. Theorem (Miyaoka). - Let $X$ be a minimal surface of general type with $c_{1}^{2}-2 c_{2}>0$, then the restriction $\mathcal{O}_{X_{1}}(1)_{\mid Z}$ is big for every horizontal irreducible 2-dimensional subvariety $Z$ of $X_{1}$.

Proof. - The Picard group of $X$ is given by

$$
\operatorname{Pic}\left(X_{1}\right)=\operatorname{Pic}(X) \oplus \mathbb{Z}[u]
$$

where $u:=\mathcal{O}_{X_{1}}(1)$, and the cohomology ring $H^{\bullet}\left(X_{1}\right)$ is given by

$$
H^{\bullet}\left(X_{1}\right)=H^{\bullet}(X)[u] /\left(u^{2}+\left(\pi^{\star} c_{1}\right) u+\pi^{\star} c_{2}\right)
$$

[ $u$ denoting rather $c_{1}\left(\mathcal{O}_{X_{1}}(1)\right)$ in that case]. In particular,

$$
u^{3}=u \cdot \pi^{\star}\left(c_{1}^{2}-c_{2}\right)=c_{1}^{2}-c_{2}, \quad u^{2} \cdot \pi^{\star} K_{X}=u \cdot \pi^{\star} c_{1}^{2}=c_{1}^{2} .
$$

Let $Z$ be an horizontal irreducible 2-dimensional subvariety. $\operatorname{In} \operatorname{Pic}\left(X_{1}\right)$, we have

$$
Z \sim m u-\pi^{\star} F
$$

for some $m>0$ and some divisor $F$ on $X$. In order to study $\mathcal{O}_{X_{1}}(1)_{\mid Z}$, we compute the Hilbert polynomial of this bundle. The coefficient of the leading term is

$$
\left(u_{\mid Z}\right)^{2}=u^{2} \cdot\left(m u-\pi^{\star} F\right)=m\left(c_{1}^{2}-c_{2}\right)+c_{1} \cdot F,
$$

by the above Chern class relations. The main difficulty is to control the term $c_{1} \cdot F$. For this, the idea is to use a semi-stability inequality. The multiplication morphism by the canonical section of $\mathcal{O}(Z)$ defines a sheaf injection $\mathcal{O}\left(\pi^{\star} F\right) \hookrightarrow \mathcal{O}_{X_{1}}(m)$. By taking the direct images on $X$, we get

$$
\mathcal{O}(F) \hookrightarrow \pi_{\star} \mathcal{O}_{X_{1}}(m)=S^{m} T_{X}^{\star}
$$


Using the $K_{X}$-semi-stability of $T_{X}^{\star}$ (see [Yau78] or [Bo79]), we infer

$$
F \cdot\left(-c_{1}\right) \leq \frac{c_{1}\left(S^{m} T_{X}^{\star}\right) \cdot\left(-c_{1}\right)}{m+1}=\frac{m}{2} c_{1}^{2}
$$

From $(\dagger)$, we get

$$
\left(u_{\mid Z}\right)^{2} \geq \frac{m}{2}\left(c_{1}^{2}-2 c_{2}\right)>0
$$

and Riemann-Roch implies that either $\mathcal{O}_{X_{1}}(1)_{\mid Z}$ or $\mathcal{O}_{X_{1}}(-1)_{\mid Z}$ is big. To decide for the sign, we observe that $K_{X}$ is big and nef and compute

$$
u_{\mid Z} \cdot \pi^{\star} K_{X}=u \cdot\left(m u-\pi^{\star} F\right) \cdot\left(-c_{1}\right)=m c_{1}^{2}+c_{1} \cdot F
$$

from this we get $u_{\mid Z} \cdot \pi^{\star} K_{X} \geq \frac{m}{2} c_{1}^{2}>0$ by the semi-stability inequality. It follows that $\mathcal{O}_{X_{1}}(1)_{\mid Z}$ is big.

By applying the above theorem of Miyaoka to the horizontal components $Z$ of $B_{1}$, we infer as in Theorem 1.2 that every nonconstant entire curve $f: \mathbb{C} \rightarrow X$ is contained in the base locus of $\mathcal{O}_{X_{1}}(k) \otimes \mathcal{O}(-A)_{\mid Z}$ for $k$ large, if $A$ is a given ample divisor. Therefore $f$ is algebraically degenerate.

2.4 Remark. - Unfortunately, the "order 1" techniques developped in this section are insufficient to deal with surfaces in $\mathbb{P}^{3}$, because in this case

$$
c_{1}^{2}=d(d-4)^{2}<c_{2}=d\left(d^{2}-4 d+6\right) .
$$

Lemma 3.4 below shows in fact that $H^{0}\left(X, S^{m} T_{X}^{\star}\right)=0$ for all $m>0$.

\section{Base locus of 2-jets}

The theory of directed manifolds and Semple jet bundles makes it possible to extend the techniques of section 2 to the case of higher order jets. The existence of suitable algebraic foliations is provided by the following simple observation, once sufficient information on the base locus $B_{k}$ is known.

3.1. Lemma. - Let $\left(X_{k}, V_{k}\right)$ be the bundle of projectivized $k$-jets associated with a surface $X$ and $V=T_{X}$. For any irreducible "horizontal hypersurface" $Z \subset X_{k}$ (i.e. such that $\pi_{k, k-1}(Z)=X_{k-1}$ ), the intersection $T_{Z} \cap V_{k}$ defines a distribution of lines on a Zariski open subset of $Z$, thus inducing a (possibly singular) 1-dimensional foliation on a desingularization of $Z$.

Proof. — We have rank $V_{k}=2$ and an exact sequence

$$
0 \longrightarrow T_{X_{k} / X_{k-1}} \longrightarrow V_{k} \longrightarrow \mathcal{O}_{X_{k}}(-1) \longrightarrow 0
$$

which follows directly from the inductive definition of $V_{k}$. Thus the intersection $T_{Z} \cap V_{k}$ defines a distribution of lines on the Zariski open subset of $Z$ equal to the set of regular points at which $\pi_{k, k-1}: Z \rightarrow X_{k-1}$ is étale (at such points, $V_{k}$ contains the vertical direction and $T_{Z}$ does not, thus $V_{k}$ and $T_{Z}$ are transverse). 
For general order $k$, it is hard to get a simple decomposition of the jet bundles $E_{k, m} T_{X}^{\star}$, and thus to calculate their Euler characteristic. However, for $k=2$ and $\operatorname{dim} X=2$, it is observed in [Dem95] that one has the remarkably simple filtration

$$
\mathrm{Gr}^{\bullet} E_{2, m} T_{X}^{\star}=\bigoplus_{0 \leq j \leq m / 3} S^{m-3 j} T_{X}^{\star} \otimes K_{X}^{\otimes j} .
$$

An elementary interpretation of this filtration consists in writing an invariant polynomial differential operator as

$$
Q(f)=\sum_{0 \leq j \leq m / 3} \sum_{\alpha \in \mathbb{N}^{2},|\alpha|=m-3 j} a_{\alpha, j}(f)\left(f^{\prime}\right)^{\alpha}\left(f^{\prime} \wedge f^{\prime \prime}\right)^{j}
$$

where

$$
f=\left(f_{1}, f_{2}\right), \quad\left(f^{\prime}\right)^{\alpha}=\left(f_{1}^{\prime}\right)^{\alpha_{1}}\left(f_{2}^{\prime}\right)^{\alpha_{2}}, \quad f^{\prime} \wedge f^{\prime \prime}=f_{1}^{\prime} f_{2}^{\prime \prime}-f_{1}^{\prime \prime} f_{2}^{\prime} .
$$

As suggested by Green-Griffiths [GG80], we use the Riemann-Roch formula to derive an existence criterion for global jet differentials. A calculation based on the above filtration of $E_{2, m} T_{X}^{\star}$ yields

$$
\chi\left(X, E_{2, m} T_{X}^{\star}\right)=\frac{m^{4}}{648}\left(13 c_{1}^{2}-9 c_{2}\right)+O\left(m^{3}\right) .
$$

On the other hand,

$$
H^{2}\left(X, E_{2, m} \otimes \mathcal{O}(-A)\right)=H^{0}\left(X, K_{X} \otimes E_{2, m} T_{X}^{\star} \otimes \mathcal{O}(A)\right)
$$

by Serre duality. Since $K_{X} \otimes\left(E_{2, m} T_{X}^{\star}\right) \otimes \mathcal{O}(A)$ admits a filtration with graded pieces

$$
S^{m-3 j} T_{X} \otimes K_{X}^{\otimes 1-j} \otimes \mathcal{O}(A),
$$

and $h^{0}\left(X, S^{p} T_{X} \otimes K_{X}^{\otimes q}\right)=0, p-2 q>0$, by Bogomolov's vanishing theorem on the general type surface $X$, we find

$$
h^{2}\left(X, E_{2, m} T_{X}^{\star} \otimes \mathcal{O}(-A)\right)=0
$$

for $m$ large. In the special case when $X$ is a smooth surface of degree $d$ in $\mathbb{P}_{\mathbb{C}}^{3}$, we take $A=\mathcal{O}(1)_{\mid X}$. Then we have $c_{1}=(4-d) h$ and $c_{2}=\left(d^{2}-4 d+6\right) h^{2}$ where $h=c_{1}\left(\mathcal{O}(1)_{\mid X}\right), h^{2}=d$, thus

$$
\chi\left(E_{2, m} T_{X}^{\star} \otimes \mathcal{O}(-A)\right)=d\left(4 d^{2}-68 d+154\right) \frac{m^{4}}{648}+O\left(m^{3}\right) .
$$

A straightforward computation shows that the leading coefficient $4 d^{2}-68 d+154$ is positive if $d \geq 15$, and a count of degrees implies that the $H^{2}$ group vanishes whenever $((m-3 j)+2(j-1))(d-4)-1>0$ for all $j \leq m / 3$. For this, it is enough that $2(m / 3-1)(d-4)-1>0$, which is the case for instance if $d \geq 5$ and $m \geq 5$. Consequently we get the following

3.2. Theorem ([Dem95]). - If $X$ is an algebraic surface of general type and $A$ an ample line bundle over $X$, then

$$
h^{0}\left(X, E_{2, m} T_{X}^{\star} \otimes \mathcal{O}(-A)\right) \geq \frac{m^{4}}{648}\left(13 c_{1}^{2}-9 c_{2}\right)-O\left(m^{3}\right) .
$$


In particular:

(a) If $13 c_{1}^{2}-9 c_{2}>0$, then $\theta_{2}<0$.

(b) Every smooth surface $X \subset \mathbb{P}^{3}$ of degree $d \geq 15$ has $\theta_{2}<0$.

We now recall a few basic facts from [Dem95]. As $X_{2} \rightarrow X_{1} \rightarrow X$ is a tower of $\mathbb{P}^{1}$-bundles over $X$, the Picard group $\operatorname{Pic}\left(X_{2}\right)=\operatorname{Pic}(X) \oplus \mathbb{Z} u_{1} \oplus \mathbb{Z} u_{2}$ consists of all isomorphism classes of line bundles

$$
\pi_{2,1}^{\star} \mathcal{O}_{X_{1}}\left(a_{1}\right) \otimes \mathcal{O}_{X_{2}}\left(a_{2}\right) \otimes \pi_{2,0}^{\star} L
$$

where $L \in \operatorname{Pic}(X)$. For simplicity of notation, we set

$$
\begin{aligned}
& u_{1}=\pi_{2,1}^{\star} \mathcal{O}_{X_{1}}(1), \quad u_{2}=\mathcal{O}_{X_{2}}(1), \\
& \mathcal{O}_{X_{2}}\left(a_{1}, a_{2}\right):=\pi_{2,1}^{\star} \mathcal{O}_{X_{1}}\left(a_{1}\right) \otimes \mathcal{O}_{X_{2}}\left(a_{2}\right)
\end{aligned}
$$

for any pair of integers $\left(a_{1}, a_{2}\right) \in \mathbb{Z}^{2}$. The canonical injection $\mathcal{O}_{X_{2}}(-1) \hookrightarrow \pi_{2}^{\star} V_{1}$ and the exact sequence

$$
0 \longrightarrow T_{X_{1} / X} \longrightarrow V_{1} \stackrel{\left(\pi_{1}\right)_{\leftarrow}}{\longrightarrow} \mathcal{O}_{X_{1}}(-1) \longrightarrow 0
$$

yield a canonical line bundle morphism

$$
\mathcal{O}_{X_{2}}(-1) \stackrel{\left(\pi_{2}^{\star}\right) \circ\left(\pi_{1}\right)_{\star}}{\longrightarrow} \pi_{2}^{\star} \mathcal{O}_{X_{1}}(-1)
$$

which admits precisely the hyperplane section $D_{2}:=P\left(T_{X_{1} / X}\right) \subset X_{2}=P\left(V_{1}\right)$ as its zero divisor. Hence we find $\mathcal{O}_{X_{2}}(-1)=\pi_{2}^{\star} \mathcal{O}_{X_{1}}(-1) \otimes \mathcal{O}\left(-D_{2}\right)$ and

$$
\mathcal{O}_{X_{2}}(-1,1) \simeq \mathcal{O}\left(D_{2}\right)
$$

is associated with an effective divisor in $X_{2}$.

3.3. Lemma. - With respect to the projection $\pi_{2,0}: X_{2} \rightarrow X$, the weighted line bundle $\mathcal{O}_{X_{2}}\left(a_{1}, a_{2}\right)$ is

(a) relatively effective if and only if $a_{1}+a_{2} \geq 0$ and $a_{2} \geq 0$;

$\left(\mathrm{a}^{\prime}\right)$ relatively big if and only if $a_{1}+a_{2}>0$ and $a_{2}>0$;

(b) relatively nef if and only if $a_{1} \geq 2 a_{2} \geq 0$;

$\left(\mathrm{b}^{\prime}\right)$ relatively ample if and only if $a_{1}>2 a_{2}>0$.

Moreover, the following properties hold.

(c) For $m=a_{1}+a_{2} \geq 0$, there is an injection

$$
\left(\pi_{2,0}\right)_{\star}\left(\mathcal{O}_{X_{2}}\left(a_{1}, a_{2}\right)\right) \hookrightarrow E_{2, m} T_{X}^{\star},
$$

and the injection is an isomorphism if $a_{1}-2 a_{2} \leq 0$.

(d) Let $Z \subset X_{2}$ be an irreducible divisor such that $Z \neq D_{2}$. Then in $\operatorname{Pic}\left(X_{2}\right)$ we have

$$
Z \sim a_{1} u_{1}+a_{2} u_{2}+\pi_{2,0}^{\star} L, \quad L \in \operatorname{Pic}(X)
$$


where $a_{1} \geq 2 a_{2} \geq 0$.

(e) Let $F \in \operatorname{Pic}(X)$ be any divisor or line bundle. In $H^{\bullet}\left(X_{2}\right)=H^{\bullet}(X)\left[u_{1}, u_{2}\right]$, we have the intersection equalities

$$
\begin{aligned}
& u_{1}^{4}=0, \quad u_{1}^{3} u_{2}=c_{1}^{2}-c_{2}, \quad u_{1}^{2} u_{2}^{2}=c_{2}, \quad u_{1} u_{2}^{3}=c_{1}^{2}-3 c_{2}, \quad u_{2}^{4}=5 c_{2}-c_{1}^{2}, \\
& u_{1}^{3} \cdot F=0, \quad u_{1}^{2} u_{2} \cdot F=-c_{1} \cdot F, \quad u_{1} u_{2}^{2} \cdot F=0, \quad u_{2}^{3} \cdot F=0 .
\end{aligned}
$$

Proof. - The exact sequence defining $V_{1}$ shows that $V_{1}$ has splitting type

$$
V_{1 \mid F_{1}}=\mathcal{O}(2) \oplus \mathcal{O}(-1)
$$

along the fibers $F_{1} \simeq \mathbb{P}^{1}$ of $X_{1} \rightarrow X$, since $T_{X_{1} / X \mid F_{1}}=\mathcal{O}(2)$. Hence the fibers $F_{2}$ of $X_{2} \rightarrow X$ are Hirzebruch surfaces $P(\mathcal{O}(2) \oplus \mathcal{O}(-1)) \simeq P(\mathcal{O} \oplus \mathcal{O}(-3))$ and

$$
\mathcal{O}_{X_{2}}(1)_{\mid F_{2}}=\mathcal{O}_{P(\mathcal{O}(2) \oplus \mathcal{O}(-1))}(1) \text {. }
$$

It is clear that the condition $a_{2} \geq 0$ is necessary for $\mathcal{O}_{X_{2}}\left(a_{1}, a_{2}\right)_{\mid F_{2}}$ to be nef or to have nontrivial sections. In that case, by taking the direct image by $\pi_{2,1}: X_{2} \rightarrow X_{1}$, global sections of $\mathcal{O}_{X_{2}}\left(a_{1}, a_{2}\right)_{\mid F_{2}}$ can be viewed as global sections over $F_{1} \simeq \mathbb{P}^{1}$ of

$$
S^{a_{2}}(\mathcal{O}(-2) \oplus \mathcal{O}(1)) \otimes \mathcal{O}\left(a_{1}\right)=\bigoplus_{0 \leq j \leq a_{2}} \mathcal{O}\left(a_{1}+a_{2}-3 j\right) .
$$

The extreme terms of the summation are $\mathcal{O}\left(a_{1}+a_{2}\right)$ and $\mathcal{O}\left(a_{1}-2 a_{2}\right)$. Claims (a)-(b) follow easily from this, and $(\mathrm{a})^{\prime},(\mathrm{b})^{\prime}$ are also clear since "being big" or "being ample" is an open condition in $\operatorname{Pic}\left(X_{2}\right)$.

(c) We have $\mathcal{O}_{X_{2}}\left(a_{1}, a_{2}\right)=\mathcal{O}_{X_{2}}(m) \otimes \mathcal{O}\left(-a_{1} D_{2}\right)$, thus $\mathcal{O}_{X_{2}}\left(a_{1}, a_{2}\right) \subset \mathcal{O}_{X_{2}}(m)$ if $a_{1} \geq 0$ and $\mathcal{O}_{X_{2}}\left(a_{1}, a_{2}\right) \supset \mathcal{O}_{X_{2}}(m)$ if $a_{1} \leq 0$. In the first case, it is immediately clear that we get an injection

$$
\left(\pi_{2,0}\right)_{\star} \mathcal{O}\left(a_{1}, a_{2}\right) \subset\left(\pi_{2,0}\right)_{\star} \mathcal{O}_{X_{2}}(m) \stackrel{\simeq}{\longrightarrow} E_{2, m} T_{X}^{\star} .
$$

In the second case, we a priori have

$$
\left(\pi_{2,0}\right)_{\star} \mathcal{O}\left(a_{1}, a_{2}\right) \supset\left(\pi_{2,0}\right)_{\star} \mathcal{O}_{X_{2}}(m) \stackrel{\simeq}{\longrightarrow} E_{2, m} T_{X}^{\star},
$$

but the above splitting formula shows that $\left(\pi_{2,0}\right)_{\star} \mathcal{O}\left(a_{1}, a_{2}\right)$ is already largest possible when $a_{1}-2 a_{2} \leq 0$ (which is the case e.g. if $\left(a_{1}, a_{2}\right)=(0, m)$ ). Hence we have an isomorphism in that case.

(d) If $a_{1}<2 a_{2}$, we have an injection

$$
\mathcal{O}_{X_{2}}\left(a_{1}+1, a_{2}-1\right)=\mathcal{O}_{X_{2}}\left(a_{1}, a_{2}\right) \otimes \mathcal{O}_{X_{2}}\left(-D_{2}\right) \subset \mathcal{O}_{X_{2}}\left(a_{1}, a_{2}\right)
$$

which induces the same space of sections over each fibre $F_{2}$. This shows that every divisor $Z$ in the linear system $\left|\mathcal{O}_{X_{2}}\left(a_{1}, a_{2}\right) \otimes \pi_{2,0}^{\star} L\right|$ contains $D_{2}$ as an irreducible component, and therefore cannot be irreducible unless $Z=D_{2}$.

(e) More general calculations are made in [Dem95]. Our formulas are easy consequences of the relations $u_{1}^{2}+c_{1} u_{1}+c_{2}=0$ and $u_{2}^{2}+c_{1}\left(V_{1}\right) u_{2}+c_{2}\left(V_{1}\right)=0$, where

$$
c_{1}\left(V_{1}\right)=c_{1}+u_{1}, \quad c_{2}\left(V_{1}\right)=c_{2}-u_{1}^{2}=2 c_{2}+c_{1} u_{1} .
$$


Under the condition $13 c_{1}^{2}-9 c_{2}>0$, Theorem 3.2 shows that the order 2 base locus $B_{2}$ is a proper algebraic subset of $X_{2}$. In order to improve Miyaoka's result 2.3, we are going to study the restriction of the line bundle $\mathcal{O}_{X_{2}}(1)$ to any 3 -dimensional component of $B_{2}$ (if such components exist). We get the following

3.4. Proposition. - Let $X$ be a minimal surface of general type. If $c_{1}^{2}-\frac{9}{7} c_{2}>0$, then the restriction of $\mathcal{O}_{X_{2}}(1)$ to every irreducible 3-dimensional component $Z$ of $B_{2} \subset X_{2}$ which projects onto $X_{1}$ ("horizontal component") and differs from $D_{2}$ is big.

Proof. - Write

$$
Z \sim a_{1} u_{1}+a_{2} u_{2}-\pi_{2,0}^{\star} F, \quad\left(a_{1}, a_{2}\right) \in \mathbb{Z}^{2}, \quad a_{1} \geq 2 a_{2}>0,
$$

where $F$ is some divisor in $X$. Our strategy is to show that $\mathcal{O}_{X_{2}}(2,1)_{\mid Z}$ is big. By Lemma 3.3 (e), we find

$$
\left(2 u_{1}+u_{2}\right)^{3} \cdot Z=\left(a_{1}+a_{2}\right)\left(13 c_{1}^{2}-9 c_{2}\right)+12 c_{1} \cdot F .
$$

Now, the multiplication morphism by the canonical section of $\mathcal{O}(Z)$ defines a sheaf injection

$$
\mathcal{O}\left(\pi_{2,0}^{\star} F\right) \hookrightarrow \mathcal{O}_{X_{2}}\left(a_{1}, a_{2}\right) .
$$

By taking direct images onto $X, \mathcal{O}(F)$ can thus be viewed as a subsheaf of

$$
\left(\pi_{2,0}\right)_{\star}\left(\mathcal{O}_{X_{2}}\left(a_{1}, a_{2}\right)\right) \subset E_{2, m} T_{X}^{\star}
$$

where $m=a_{1}+a_{2}$. Looking at the filtration of $E_{2, m} T_{X}^{\star}$, we infer that there is a nontrivial morphism

$$
\mathcal{O}(F) \hookrightarrow S^{m-3 j} T_{X}^{\star} \otimes K_{X}^{\otimes j}
$$

for some $j \leq \frac{m}{3}$. As in $\S 2$, the semistability inequality implies

$$
F \cdot K_{X} \leq\left(\frac{m-3 j}{2}+j\right) K_{X}^{2} \leq \frac{m}{2} c_{1}^{2}, \quad \text { thus } \quad-c_{1} \cdot F \leq \frac{m}{2} c_{1}^{2} .
$$

Formula ( $\dagger \dagger \dagger)$ combined with the assumption $7 c_{1}^{2}-9 c_{2}>0$ implies

$$
\left(2 u_{1}+u_{2}\right)^{3} \cdot Z \geq m\left(7 c_{1}^{2}-9 c_{2}\right)>0 .
$$

The latter inequality still holds if we replace $\mathcal{O}_{X_{2}}(2,1)$ by $\mathcal{O}_{X_{2}}(2+\varepsilon, 1)$ with a fixed sufficiently small positive rational number $\varepsilon$. By Riemann-Roch, either

$$
h^{0}\left(Z, \mathcal{O}_{X_{2}}((2+\varepsilon) p, p)_{\mid Z}\right) \quad \text { or } \quad h^{2}\left(Z, \mathcal{O}_{X_{2}}((2+\varepsilon) p, p)_{\mid Z}\right)
$$

grows fast as $p$ goes to infinity. We want to exclude the second possibility. For this, we look at the exact sequence

$$
0 \rightarrow \mathcal{O}(-Z) \otimes \mathcal{O}_{X_{2}}((2+\varepsilon) p, p) \rightarrow \mathcal{O}_{X_{2}}((2+\varepsilon) p, p) \rightarrow \mathcal{O}_{Z} \otimes \mathcal{O}_{X_{2}}((2+\varepsilon) p, p) \rightarrow 0
$$

and take the direct images to $X$ by the Leray spectral sequence of the fibration $X_{2} \rightarrow X$. As $\mathcal{O}_{X_{2}}(2+\varepsilon, 1)$ is relatively ample, the higher $R^{q}$ sheaves are zero and we see immediately that

$$
\begin{aligned}
h^{2}\left(Z, \mathcal{O}_{X_{2}}((2+\varepsilon) p, p)_{\mid Z}\right) \leq & h^{2}\left(X_{2}, \mathcal{O}_{X_{2}}((2+\varepsilon) p, p)\right) \\
& +h^{3}\left(X_{2}, \mathcal{O}_{X_{2}}(-Z) \otimes \mathcal{O}_{X_{2}}((2+\varepsilon) p, p)\right) \\
\leq & h^{2}\left(X,\left(\pi_{2,0}\right)_{\star} \mathcal{O}_{X_{2}}((2+\varepsilon) p, p)\right)
\end{aligned}
$$


By Bogomolov's vanishing theorem, the latter group is zero. Thus, we obtain that $\mathcal{O}_{X_{2}}(2+\varepsilon, 1)_{\mid Z}$ is big, and this implies that $\mathcal{O}_{X_{2}}(1)_{\mid Z}$ is also big because we have a sheaf injection

$$
\mathcal{O}_{X_{2}}(2+\varepsilon, 1)=\mathcal{O}_{X_{2}}(3+\varepsilon) \otimes \mathcal{O}\left(-(2+\varepsilon) D_{2}\right) \longleftrightarrow \mathcal{O}_{X_{2}}(3+\varepsilon)
$$

(if necessary, pass to suitable tensor multiples to avoid denominators).

3.5. Corollary. - Let $X$ be a surface of general type such that $c_{1}^{2}-\frac{9}{7} c_{2}>0$. Then the irreducible components of the Green-Griffiths locus $B_{2} \subset X_{2}$ are of dimension 2 at most, except for the divisor $D_{2} \subset X_{2}$.

This corollary is not really convincing, since we already have sections in $H^{0}\left(X, S^{m} T_{X}^{\star} \otimes \mathcal{O}(-A)\right)$ under the weaker condition $c_{1}^{2}-c_{2}>0$ (a condition which is anyhow too restrictive to encompass the case of surfaces in $\mathbb{P}^{3}$ ). Fortunately, under the additional assumption that the surface has Picard group $\mathbb{Z}$, one can get a more precise inequality than the stability inequality, and that inequality turns out to be sufficient to treat the case of generic surfaces of sufficiently high degree in $\mathbb{P}^{3}$.

\section{Proof of the Main Theorem}

We assume here throughout that $X$ is a surface of general type such that $\operatorname{Pic}(X)=\mathbb{Z}$. Then the canonical bundle $K_{X}$ is ample, and we have $c_{1}^{2}>0, c_{2} \geq 0$. Our first goal is to estimate the 2 -jet threshold of $X$. Consider a non trivial section

$$
\sigma \in H^{0}\left(X, E_{2, m} T_{X}^{\star} \otimes \mathcal{O}\left(t K_{X}\right)\right), \quad m>0, \quad t \in \mathbb{Q}
$$

and its zero divisor

$$
Z_{\sigma}=m u_{2}+t \pi_{2,0}^{\star} K_{X} \quad \text { in } \operatorname{Pic}\left(X_{2}\right) .
$$

Let $Z_{\sigma}=\sum p_{j} Z_{j}$ be the decomposition of $Z_{\sigma}$ in irreducible components. From the equality $\operatorname{Pic}\left(X_{2}\right)=\operatorname{Pic}(X) \oplus \mathbb{Z} u_{1} \oplus \mathbb{Z} u_{2}$ and the assumption $\operatorname{Pic}(X) \simeq \mathbb{Z}$, we find

$$
Z_{j} \sim a_{1, j} u_{1}+a_{2, j} u_{2}+t_{j} \pi_{2,0}^{\star} K_{X}
$$

for suitable integers $a_{1, j}, a_{2, j} \in \mathbb{Z}$ and rational numbers $t_{j} \in \mathbb{Q}$. By Lemma 3.3, as $Z_{j}$ is effective, we must have one of the following three disjoint cases:

- $\left(a_{1, j}, a_{2, j}\right)=(0,0)$ and $Z_{j} \in \pi_{2,0}^{\star} \operatorname{Pic}(X), t_{j}>0$;

- $\left(a_{1, j}, a_{2, j}\right)=(-1,1)$, then $Z_{j}$ contains $D_{2}$, so $Z_{j}=D_{2}$ and $t_{j}=0$;

- $a_{1, j} \geq 2 a_{2, j} \geq 0$ and $m_{j}:=a_{1, j}+a_{2, j}>0$.

In the third case, we obtain a section

$$
\sigma_{j} \in H^{0}\left(X_{2}, \mathcal{O}_{X_{2}}\left(m_{j}\right) \otimes \pi_{2,0}^{\star} \mathcal{O}\left(t_{j} K_{X}\right)\right)
$$

whose divisor is $Z_{j}+a_{1, j} D_{2}$. As $m=\sum m_{j}$ and $t=\sum t_{j}$, it is clear that $\frac{t}{m} \geq \min \frac{t_{j}}{m_{j}}$, where the minimum is taken over those sections arising from the 
third case. It follows that the 2-jet threshold can be computed by using only those sections which correspond to an irreducible divisor (regardless of $D_{2}$ which is "negligible" in this matter). We use the following lemma.

4.1. Lemma. - Let $m=3 p+q, 0 \leq q \leq 2$ a positive integer.

(a) There are bundle morphisms

$$
E_{2, m} T_{X}^{\star} \rightarrow E_{2, m-3} T_{X}^{\star} \otimes K_{X} \rightarrow E_{2, m-6} T_{X}^{\star} \otimes K_{X}^{2} \rightarrow \cdots \rightarrow S^{q} T_{X}^{\star} \otimes K_{X}^{p} .
$$

(b) There is a (nonlinear!) discriminant mapping

$$
\Delta: E_{2, m} T_{X}^{\star} \rightarrow S^{(p-1)(3 p+2 q)} T_{X}^{\star} \otimes K_{X}^{p(p-1)} .
$$

(c) If $\theta_{1} \geq 0$, the 2-jet threshold satisfies

$$
\theta_{2} \geq \min \left(\theta_{2,3}, \theta_{2,4}, \theta_{2,5}, \frac{1}{2} \theta_{1}-\frac{1}{6}\right)
$$

Proof. - (a) is a consequence of the filtration described earlier. In order to prove (b), we write an element of $E_{2, m} T_{X}^{\star}$ in the form

$$
P(f)=\sum_{0 \leq j \leq p} a_{j} \cdot f^{\prime 3(p-j)+q} W^{j}
$$

where the $a_{j}$ is viewed as an element of $S^{3(p-j)+q} T_{X}^{\star} \otimes K_{X}^{j}$, and

$$
W=f_{1}^{\prime} f_{2}^{\prime \prime}-f_{1}^{\prime \prime} f_{2}^{\prime} \in \Lambda^{2} T_{X}=K_{X}^{-1} .
$$

The discriminant $\Delta(P)$ is calculated by interpreting $P$ as a polynomial in the indeterminate $W$. The precise formula is

$$
\left.\Delta(P)=\frac{1}{a_{p}} \mid \begin{array}{cccccccccc}
a_{0} & a_{1} & a_{2} & a_{3} & \ldots & a_{p-1} & a_{p} & 0 & 0 & \ldots \\
0 & a_{0} & a_{1} & a_{2} & a_{3} & \ldots & a_{p-1} & a_{p} & 0 & \ldots \\
\vdots & & & & & & & & & \vdots \\
\ldots & 0 & 0 & a_{0} & a_{1} & a_{2} & \ldots & a_{p-1} & a_{p} & 0 \\
\ldots & 0 & 0 & 0 & a_{0} & a_{1} & a_{2} & \ldots & a_{p-1} & a_{p} \\
b_{0} & b_{1} & b_{2} & \ldots & b_{p-2} & b_{p-1} & 0 & 0 & 0 & \ldots \\
0 & b_{0} & b_{1} & b_{2} & \ldots & b_{p-2} & b_{p-1} & 0 & 0 & \ldots \\
\vdots & & & & & & & & & \vdots \\
\ldots & 0 & 0 & 0 & b_{0} & b_{1} & \ldots & b_{p-2} & b_{p-1} & 0 \\
\ldots & 0 & 0 & 0 & 0 & b_{0} & b_{1} & \ldots & b_{p-2} & b_{p-1}
\end{array}\right\} p-1
$$

where

$$
\frac{\partial P}{\partial W}=\sum_{0 \leq j \leq p-1} b_{j} W^{j}=\sum_{0 \leq j \leq p-1}(j+1) a_{j+1} W^{j}
$$

is the derived polynomial. By counting the degrees of all terms $a_{j}$ and $b_{j}$ as polynomials in $f^{\prime}$, one sees that $\Delta(P)$ is a homogeneous polynomial. Its degree is equal to that of the diagonal term

$$
\frac{1}{a_{p}} a_{0}^{p-1} b_{p-1}^{p}=\operatorname{Const}\left(a_{0} a_{p}\right)^{p-1}
$$


which lives in $S^{(p-1)(3 p+2 q)} T_{X}^{\star} \otimes K_{X}^{p(p-1)}$. Geometrically, if $P$ is a germ of section of $E_{2, m} T_{X}^{\star},(P=0)$ defines a germ of divisor $Z \subset X_{2}$, and $\Delta(P)=0$ is the divisor in $X_{1}$ along which the projection $Z \rightarrow X_{1}$ has branched points.

(c) By the observations made at the beginning of the section, we can start with a section in

$$
\sigma \in H^{0}\left(X, E_{2, m} T_{X}^{\star} \otimes \mathcal{O}\left(t K_{X}\right)\right)
$$

associated with an irreducible divisor $Z$ in $X_{2}$ (up to some $D_{2}$ components). If $m=1,2$, we have $E_{2, m} T_{X}^{\star}=S^{m} T_{X}^{\star}$, thus $\frac{t}{m} \geq \theta_{1} \geq \frac{1}{2} \theta_{1}-\frac{1}{6}$. If $m=3,4,5$, then

$$
\frac{t}{m} \geq \min \left(\theta_{2,3}, \theta_{2,4}, \theta_{2,5}\right)
$$

by definition. If $m=3 p+q \geq 6, p \geq 2$, we get a non trivial discriminant section

$$
\Delta \in H^{0}\left(X, S^{(p-1)(3 p+2 q)} T_{X}^{\star} \otimes \mathcal{O}\left(p(p-1) K_{X}+(2 p-2) t K_{X}\right)\right) .
$$

Therefore $2(p-1) t+p(p-1) \geq(p-1)(3 p+2 q) \theta_{1}$, and this implies

$$
\frac{t}{m} \geq \frac{3 p+2 q}{2 m} \theta_{1}-\frac{p}{2 m} \geq \frac{1}{2} \theta_{1}-\frac{1}{6} \text {. }
$$

Inequality (c) is proved.

Proof of the Main Theorem. If $\theta_{1}<0$, then $\mathcal{O}_{X_{1}}(1)$ is big and we conclude by a direct application of Theorem 1.2. Assume now that $X$ satisfies assumptions (a), (b), (c) of the Main Theorem. As $\theta_{2}<0$ by (a), we have a non trivial section

$$
\sigma \in H^{0}\left(X, E_{2, m} T_{X}^{\star} \otimes \mathcal{O}\left(t K_{X}\right)\right), \quad m>0, \quad t \in \mathbb{Q}, \quad t<0,
$$

and the discussion made at the beginning of the section shows that we can assume that $Z_{\sigma}=Z+a_{1} D_{2}$ for some irreducible divisor $Z$ in $X_{2}$ such that

$$
Z \sim a_{1} u_{1}+a_{2} u_{2}+t \pi_{2,0}^{\star} K_{X} \quad \text { in } \operatorname{Pic}\left(X_{2}\right), \quad a_{1}+a_{2}=m .
$$

Formula $(\dagger \dagger \dagger)$ of section 3 gives

$$
\left(2 u_{1}+u_{2}\right)^{3} \cdot Z=m\left(13 c_{1}^{2}-9 c_{2}\right)+12 t c_{1}^{2},
$$

and by definition of $\theta_{2}$ we have $t / m \geq \theta_{2}$, hence

$$
\left(2 u_{1}+u_{2}\right)^{3} \cdot Z \geq m\left(\left(13+12 \theta_{2}\right) c_{1}^{2}-9 c_{2}\right)>0
$$

under assumption (c). As in the proof of Proposition 3.4, we conclude that the restriction $\mathcal{O}_{X_{2}}(1)_{\mid Z}$ is big. Consequently, by Theorem 1.2 (or rather, by the proof of Theorem 1.2, see [Dem95]), every nonconstant entire curve $f: \mathbb{C} \rightarrow X$ is such that $f_{[2]}(\mathbb{C})$ is contained in the base locus of $\mathcal{O}_{X_{2}}(l) \otimes \pi_{2,0}^{\star} \mathcal{O}(-A)_{\mid Z}$ for $l$ large. This base locus is at most 2-dimensional, and projects onto a proper algebraic subvariety $Y$ of $X_{1}$. Therefore $f_{[1]}(\mathbb{C})$ is contained in $Y$, and the Main Theorem is proved. 


\section{Vanishing of global 2-jet differentials of degree $3,4,5$}

This section is devoted to the proof of the generic nonexistence of certain 2-jet differentials of small degree, as required in condition (d) of the Main Theorem. We start with the easier and well-known case of symmetric differentials (see e.g. Sakai [Sa78]), which we just investigate briefly for the reader's convenience.

5.1. Lemma. - Let $X$ be a nonsingular surface of degree $d$ in $\mathbb{P}^{3}, m$ a nonnegative integer and $k \in \mathbb{Z}$. Then

(a) $H^{0}\left(X, S^{m} T_{X}^{\star} \otimes \mathcal{O}(k)\right)=0 \quad$ for all $k \leq \min (2 m-1, m-2+d)$.

(b) $H^{0}\left(X, S^{m} T_{X}^{\star} \otimes \mathcal{O}(k)\right) \simeq H^{0}\left(\mathbb{P}^{3}, S^{m} T_{\mathbb{P}^{3}}^{\star} \otimes \mathcal{O}(k)\right)$ for all $k \leq m-2+d$.

(c) For $d \geq 5, X$ is of general type and its 1-jet threshold satisfies

$$
\frac{1}{d-4} \leq \theta_{1} \leq \frac{2}{d-4}, \quad \theta_{1, m} \geq \frac{\min (2,1+(d-1) / m)}{d-4} \quad \text { for all } m>0 .
$$

Proof. — The Euler exact sequence

$$
0 \longrightarrow \mathcal{O} \longrightarrow \mathcal{O}(1)^{\oplus 4} \longrightarrow T_{\mathbb{P}^{3}} \longrightarrow 0
$$

gives an exact sequence

$0 \rightarrow S^{m} T_{\mathbb{P}^{3}}^{\star} \otimes \mathcal{O}(k) \rightarrow S^{m}\left(\mathcal{O}^{\oplus 4}\right) \otimes \mathcal{O}(k-m) \rightarrow S^{m-1}\left(\mathcal{O}^{\oplus 4}\right) \otimes \mathcal{O}(k-m+1) \rightarrow 0$.

As $H^{q}\left(\mathbb{P}^{3}, \mathcal{O}(p)\right)=0$ for all $q=1,2$ and for $q=0, p<0$, we easily conclude that $H^{q}\left(\mathbb{P}^{3}, S^{m} T_{\mathbb{P}^{3}}^{\star} \otimes \mathcal{O}(k)\right)=0$ in all cases

$$
q=0, \quad k \leq 2 m-1, \quad \text { or } \quad q=1, \quad k \leq m-2, \quad \text { or } \quad q=2, \quad k \in \mathbb{Z} .
$$

[ The case $q=0$ is obtained by considering the restriction of sections to arbitrary lines in $\mathbb{P}^{3}$, and by using $\left.T_{\mathbb{P}^{1}}^{\star}=\mathcal{O}(-2)\right]$. The exact sequence

$$
0 \longrightarrow \mathcal{O}_{\mathbb{P}^{3}}(-d) \longrightarrow \mathcal{O}_{\mathbb{P}^{3}} \longrightarrow \mathcal{O}_{X} \rightarrow 0
$$

twisted by $S_{\mathbb{P}^{3}}^{m}$ then shows that $H^{q}\left(X, S^{m} T_{\mathbb{P}^{3} \mid X}^{\star} \otimes \mathcal{O}(k)\right)=0$ for $q \leq 1$ and $k \leq m-2$, and that

$$
H^{0}\left(X, S^{m} T_{\mathbb{P}^{3} \mid X}^{\star} \otimes \mathcal{O}_{X}(k) \simeq H^{0}\left(\mathbb{P}^{3}, S^{m} T_{\mathbb{P}^{3}}^{\star} \otimes \mathcal{O}(k)\right)\right.
$$

for $k \leq m-2+d$. Finally, by taking symmetric powers in the dual sequence of

$$
0 \longrightarrow T_{X} \longrightarrow T_{\mathbb{P}^{3} \mid X} \longrightarrow \mathcal{O}_{X}(d) \longrightarrow 0,
$$

we find a sequence

$$
0 \longrightarrow S^{m-1} T_{\mathbb{P}^{3} \mid X}^{\star} \otimes \mathcal{O}_{X}(-d) \longrightarrow S^{m} T_{\mathbb{P}^{3} \mid X}^{\star} \longrightarrow S^{m} T_{X}^{\star} \longrightarrow 0,
$$

from which it readily follows that $H^{0}\left(X, S^{m} T_{X}^{\star} \otimes \mathcal{O}(k)\right) \simeq H^{0}\left(\mathbb{P}^{3}, S^{m} T_{\mathbb{P}^{3}}^{\star} \otimes \mathcal{O}(k)\right)$ for $k \leq m-2+d$. (b) is proved, and (a) is a special case.

(c) We have $K_{X}=\mathcal{O}_{X}(d-4)$. Property (a) shows that there are no nonzero sections in $H^{0}\left(X, S^{m} T_{X}^{\star} \otimes \mathcal{O}\left(t K_{X}\right)\right)$ unless $t(d-4) \geq \min (2 m, m-1+d)$, and this certainly implies $t / m>1 /(d-4)$, whence the lower bound for $\theta_{1}$. On the 
other hand, by taking $m=d-2$ and $k=2 m$, we do find a nonzero section in $H^{0}\left(X, S^{m} T_{X}^{\star} \otimes \mathcal{O}_{X}(2 m)\right)$, whence the upper bound.

We now turn ourselves to the question of the existence of 2-jet differentials of small degree. For this question, it is especially convenient to use the concept of meromorphic connections, in the spirit of the work of Y.T. Siu [Siu87] and A. Nadel [Na89]. By definition, a meromorphic connection is an operator acting on meromorphic vector fields $v=\sum v_{i} \partial / \partial z_{i}, w=\sum w_{i} \partial / \partial z_{i}$ which, in any complex coordinate system $\left(z_{1}, \ldots, z_{n}\right)$, has the form

$$
\nabla_{w} v=\sum_{1 \leq i, k \leq n}\left(w_{i} \frac{\partial v_{k}}{\partial z_{i}}+\sum_{1 \leq j \leq n} \Gamma_{i j}^{k} w_{i} v_{j}\right) \frac{\partial}{\partial z_{k}}=d_{w} v+\Gamma \cdot(w, v)
$$

The Christoffel symbols $\Gamma=\left(\Gamma_{i j}^{k}\right)_{1 \leq i, j, k \leq n}$ are thus meromorphic functions. To such a connection, we associate the Wronskian operator

$$
W_{\nabla}(f)=f^{\prime} \wedge f_{\nabla}^{\prime \prime}, \quad f^{\prime \prime}=\nabla_{f^{\prime}} f^{\prime},
$$

given explicitly in coordinates by

$$
\begin{aligned}
W_{\nabla}(f)= & \left(\left(f_{1}^{\prime} f_{2}^{\prime \prime}-f_{1}^{\prime \prime} f_{2}^{\prime}\right)-\Gamma_{1,1}^{2} f_{1}^{\prime 3}+\Gamma_{2,2}^{1} f_{2}^{\prime 3}\right. \\
& \left.+\left(\Gamma_{1,1}^{1}-\Gamma_{1,2}^{2}-\Gamma_{2,1}^{2}\right) f_{1}^{\prime 2} f_{2}^{\prime}-\left(\Gamma_{2,2}^{2}-\Gamma_{1,2}^{1}-\Gamma_{2,1}^{1}\right) f_{1}^{\prime} f_{2}^{\prime 2}\right) \frac{\partial}{\partial z_{1}} \wedge \frac{\partial}{\partial z_{2}} .
\end{aligned}
$$

If $B$ is the pole divisor of the coefficients $\Gamma_{i j}^{k}$, the Wronskian operator $W_{\nabla}(f)$ takes values in $\mathcal{O}(B) \otimes \mathcal{O}\left(\Lambda^{2} T_{X}\right)=\mathcal{O}\left(B-K_{X}\right)$, thus

$$
W_{\nabla} \in H^{0}\left(X, E_{2,3} T_{X}^{\star} \otimes \mathcal{O}\left(B-K_{X}\right)\right) .
$$

The relevant type of connections we need are the "meromorphic partial projective connections" introduced in [EG96] and [DEG97]. A meromorphic partial projective connection is a section of the quotient sheaf of the sheaf of meromorphic connections modulo meromorphic zero order operators of the form $\alpha(w) v+\beta(v) w$. The Christoffel symbols are thus supposed to be determined only up to terms of the form

$$
\widetilde{\Gamma}_{i j}^{k}-\Gamma_{i j}^{k}=\alpha_{i} \delta_{j k}+\beta_{j} \delta_{i k}
$$

Adding such terms to $\nabla$ replaces $f_{\nabla}^{\prime \prime}$ with $f_{\nabla}^{\prime \prime}+\alpha\left(f^{\prime}\right) f^{\prime}+\beta\left(f^{\prime}\right) f^{\prime}$, and thus does not change the corresponding Wronskian operator. In dimension 2, a meromorphic connection depends on 8 Christoffel symbols, but a partial projective meromorphic connection depends only on 4 Christoffel symbols. Since the Wronskian operator on a surface also depends only on 4 coefficients, we see in that case that there is a oneto-one correspondence between partial meromorphic connections and Wronskian operators, and more precisely, between partial meromorphic connections with pole divisor $\leq B$ and Wronskian operators $W \in H^{0}\left(X, E_{2,3} T_{X}^{\star} \otimes \mathcal{O}\left(B-K_{X}\right)\right)$. To make this even more precise, let us consider the exact sequences

$$
\begin{gathered}
0 \longrightarrow S^{3} T_{X}^{\star} \longrightarrow E_{2,3} T_{X}^{\star} \stackrel{\Phi}{\longrightarrow} K_{X} \longrightarrow 0 \\
0 \longrightarrow S^{3} T_{X}^{\star} \otimes \mathcal{O}\left(B-K_{X}\right) \longrightarrow E_{2,3} T_{X}^{\star} \otimes \mathcal{O}\left(B-K_{X}\right) \stackrel{\Phi}{\longrightarrow} \mathcal{O}(B) \longrightarrow 0 .
\end{gathered}
$$


To any nonzero section

$$
P \in H^{0}\left(X, E_{2,3} T_{X}^{\star} \otimes \mathcal{O}\left(B-K_{X}\right)\right)
$$

corresponds a section $\beta=\Phi(P) \in H^{0}(X, \mathcal{O}(B))$ which can be viewed as the "principal symbol" of $P$ (coefficient of $\left.f_{1}^{\prime} f_{2}^{\prime \prime}-f_{2}^{\prime} f_{1}^{\prime \prime}\right)$. If the symbol $\beta$ is nonzero, we actually get a Wronskian operator

$$
W(f)=\beta(f)^{-1} P(f)
$$

with pole divisor $\leq B$.

Our next result is a basic proportionality lemma for 2-jet differentials of degree 3, 4, 5. We are indebted to Mihai Paun [Pa99] for the observation that the proportionality lemma also holds true for degrees 4 and 5 (as a consequence, we are now able to get substantially better degree bounds than in our earlier version of the manuscript). For a polynomial differential operator $P\left(f^{\prime}, f^{\prime \prime}\right)$ of total degree $m=3,4,5$, the exponent of $\left(f_{1}^{\prime} f_{2}^{\prime \prime}-f_{2}^{\prime} f_{1}^{\prime \prime}\right)^{j}$ can only take the values $j=0,1$, and we thus get an exact sequence

$$
0 \longrightarrow S^{m} T_{X}^{\star} \longrightarrow E_{2, m} T_{X}^{\star} \stackrel{\Phi}{\longrightarrow} E_{2, m-3} T_{X}^{\star} \otimes K_{X} \rightarrow 0
$$

where $E_{2, m-3} T_{X}^{\star}=S^{m-3} T_{X}^{\star}$. Explicitly, if

$$
P=\sum_{|\alpha|=m} a_{\alpha}\left(f^{\prime}\right)^{\alpha}+\sum_{|\alpha|=m-3} b_{\alpha}\left(f^{\prime}\right)^{\alpha}\left(f_{1}^{\prime} f_{2}^{\prime \prime}-f_{2}^{\prime} f_{1}^{\prime \prime}\right),
$$

then $\beta=\Phi(P)=\sum_{|\alpha|=m-3} b_{\alpha}\left(f^{\prime}\right)^{\alpha}$.

5.2. Proportionality lemma. - Let $X$ be a nonsingular surface of general type. Then, for all sections

$$
P_{i} \in H^{0}\left(X, E_{2, m_{i}} T_{X}^{\star} \otimes \mathcal{O}_{X}\left(t_{i} K_{X}\right)\right)
$$

with $m_{i}=3,4,5$ and $t_{i} \in \mathbb{Q}_{+}, 1+t_{1}+t_{2}<\left(m_{1}+m_{2}-3\right) \theta_{1, m_{1}+m_{2}-3}$, the section

$$
\beta_{1} P_{2}-\beta_{2} P_{1} \in H^{0}\left(X, E_{2, m_{1}+m_{2}-3} T_{X}^{\star} \otimes \mathcal{O}_{X}\left(\left(1+t_{1}+t_{2}\right) K_{X}\right)\right)
$$

associated with $\beta_{i}=\Phi\left(P_{i}\right)$ vanishes.

Proof. - Consider $P=\beta_{1} P_{2}-\beta_{2} P_{1}$. This is a differential polynomial operator, and $\Phi(P)=\beta_{1} \beta_{2}-\beta_{2} \beta_{1}=0$ by construction. Hence $P$ can be viewed as a section in

$$
H^{0}\left(X, S^{m_{1}+m_{2}-3} T_{X}^{\star} \otimes \mathcal{O}\left(\left(1+t_{1}+t_{2}\right) K_{X}\right)\right) .
$$

By definition of $\theta_{1, m}$, this group vanishes if

$$
1+t_{1}+t_{2}<\left(m_{1}+m_{2}-3\right) \theta_{1, m_{1}+m_{2}-3} .
$$

In particular, a nonzero section of $H^{0}\left(X, E_{2,3} T_{X}^{\star} \otimes \mathcal{O}\left(t K_{X}\right)\right)$ can be viewed as a partial meromorphic connection with pole divisor $B \leq(1+t) K_{X}$. From this, we infer

5.3. Corollary. - Let $X$ be a nonsingular surface of general type with $\operatorname{Pic}(X)=\mathbb{Z}$. Then there exists at most one partial projective connection $\nabla$ with pole divisor $B<\frac{1}{2}\left(1+3 \theta_{1,3}\right) K_{X}$. 
Examples of partial meromorphic connections with low pole orders can be explicitly constructed by means of Nadel's technique [Na89] (see also [EG96], [DEG97] and [SY97]). In particular, one can find examples - which are however highly nongeneric - for which the ratio $B / K_{X}$ takes more or less random values in the range $] 0,1]$. By adjusting the choice of $B$ as close as possible to the upper limit $\frac{1}{2}\left(1+3 \theta_{1}\right)$, we know that the connection must be unique, and a nonexistence result follows just by taking $B$ slightly smaller than the upper limit. In this way we obtain

5.4. Proposition. - Let $X$ be a generic surface of degree $d \geq 6$ in $\mathbb{P}^{3}$. Then

$$
\theta_{2, m} \geq-\frac{1}{2 m}+\frac{2-7 / 2 m}{d-4} \quad \text { for } m=3,4,5 \text {. }
$$

Proof. - Assume that $X$ is a smooth member of a linear system of surfaces

$$
X_{\lambda}=\left\{\lambda_{0} s_{0}(z)+\lambda_{1} s_{1}(z)+\lambda_{2} s_{2}(z)+\lambda_{3} s_{3}(z)=0\right\}
$$

where $s_{0}, s_{1}, s_{2}, s_{3} \in \mathbb{C}\left[z_{0}, z_{1}, z_{2}, z_{3}\right]$ are homogeneous polynomials of degree $d$. According Nadel's method [Nad89], we solve the linear system

$$
\sum_{0 \leq k \leq 3} \widetilde{\Gamma}_{i j}^{k} \frac{\partial s_{\ell}}{\partial z_{k}}=\frac{\partial^{2} s_{\ell}}{\partial z_{i} \partial z_{j}}, \quad 0 \leq i, j, \ell \leq 3,
$$

and get in this way a homogeneous meromorphic connection of degree -1 on $\mathbb{C}^{4}$. One can check that this connection descends to a partial projective meromorphic connection $\nabla$ on $\mathbb{P}^{3}$ such that $X_{\lambda}$ is totally geodesic (see [DEG97]). Let us consider the specific example

$$
X_{a}=\left\{z_{0}^{d}+z_{1}^{d}+z_{2}^{d}+z_{3}^{d}+a z_{0}^{k_{0}} z_{1}^{k_{1}} z_{2}^{k_{2}} z_{3}^{k_{3}}=0\right\},
$$

where $k_{0}, k_{1}, k_{2}, k_{3} \geq 0$ are integers such that $\sum k_{i}=d$. We take in this case

$$
s_{0}=z_{0}^{k_{0}}\left(z_{0}^{d-k_{0}}+a z_{1}^{k_{1}} z_{2}^{k_{2}} z_{3}^{k_{3}}\right), \quad s_{i}=z_{i}^{d}, \quad i=1,2,3 .
$$

A short computation shows that $X_{a}$ is nonsingular if and only if $a^{d} \neq(-d)^{d} \prod k_{i}^{-k_{i}}$ and that the pole divisor of the connection $\nabla$ is given by

$$
B=\left\{z_{0} z_{1} z_{2} z_{3}\left(d z_{0}^{k_{1}+k_{2}+k_{3}}+a k_{0} z_{1}^{k_{1}} z_{2}^{k_{2}} z_{3}^{k_{3}}\right)=0\right\}
$$

( $B$ is just the zero divisor of the denominator of the rational functions expressing solutions $\widetilde{\Gamma}_{i j}^{k}$ of the above linear system, after these rational functions have been simplified). In particular, the ratio

$$
\frac{B}{K_{X_{a}}}=\frac{4+k_{1}+k_{2}+k_{3}}{d-4}
$$

can be taken to be $\frac{p}{d-4}$ for any integer $p$ with $4 \leq p \leq d+4$. This yields a section $P_{1} \in H^{0}\left(X, E_{2,3} T_{X}^{\star} \otimes \mathcal{O}\left(t_{1} K_{X}\right)\right)$ with $t_{1}=\frac{p}{d-4}-1$. We take $p=\left[\frac{d+3}{2}\right]$ so that

$$
\frac{1}{2}+t_{1}=\frac{3+\varepsilon / 2}{d-4} \quad \text { where } \quad \varepsilon=(d+1) \bmod 2, \quad \varepsilon \in\{0,1\} .
$$


The integer $p$ must be at least equal to 4 , thus our choice is permitted if $d \geq 6$. We claim that $X=X_{a}$ has no non trivial section in

$$
H^{0}\left(X, E_{2, m} T_{X}^{\star} \otimes \mathcal{O}\left(t K_{X}\right)\right), \quad m=m_{2}=3,4,5
$$

when $\frac{1}{2}+t<\frac{2 m-3-\varepsilon / 2}{d-4}$. Indeed, for $m_{1}=3, m_{2}=m$ and $t_{2}=t$, our choices imply

$$
1+t_{1}+t_{2}<\frac{2 m}{d-4} \leq\left(m_{1}+m_{2}-3\right) \theta_{1, m_{1}+m_{2}-3},
$$

as $\theta_{1, m} \geq \theta_{1,5} \geq \frac{2}{d-4}$ for $m \leq 5$ and $d \geq 6$. By Lemma 5.2, any non zero section $P_{2} \in H^{0}\left(X, E_{2, m} T_{X}^{\star} \otimes \mathcal{O}\left(t K_{X}\right)\right)$ would yield a meromorphic connection associated with a Wronskian operator $P_{2} / \beta_{2}=P_{1} / \beta_{1}$. As $P_{1} / \beta_{1}$ is an irreducible fraction with $\operatorname{div} \beta_{1}=B$, we conclude that $\beta_{2} / \beta_{1} \in H^{0}\left(X, S^{m-3} T_{X}^{\star} \otimes \mathcal{O}\left(\left(t_{2}-t_{1}\right) K_{X}\right)\right)$ must be holomorphic, hence

$$
t_{2} \geq t_{1}+(m-3) \theta_{1, m-3} \geq t_{1}+\frac{2 m-6}{d-4} .
$$

On the other hand

$$
t_{2}=t<-\frac{1}{2}+\frac{2 m-3-\varepsilon / 2}{d-4}=t_{1}+\frac{2 m-6-\varepsilon}{d-4},
$$

contradiction. By the Zariski semicontinuity of cohomology, the group

$$
H^{0}\left(X, E_{2, m} T_{X}^{\star} \otimes \mathcal{O}\left(t K_{X}\right)\right)
$$

vanishes for a generic surface $X$, unless

$$
\frac{t}{m} \geq-\frac{1}{2 m}+\frac{2-(3+\varepsilon / 2) / m}{d-4}
$$

Proposition 5.4 is proved.

\section{McQuillan's work on algebraic foliations}

Recently, using Miyaoka's semi-positivity result for cotangent bundles of nonuniruled projective varieties [Mi87] and a dynamic diophantine approximation, McQuillan [McQ97] derived strong Nevanlinna Second Main Theorems for holomorphic mappings $f: \mathbb{C} \rightarrow X$ tangent to the leaves of an algebraic foliation.

6.1. Theorem (McQuillan). - Every parabolic leaf of an algebraic (multi-) foliation on a surface $X$ of general type is algebraically degenerate.

The assumption $c_{1}^{2}>c_{2}$ guarantees the existence of an algebraic multifoliation such that every $f: \mathbb{C} \rightarrow X$ is contained in one of the leaves. Thus McQuillan's theorem implies

6.2. Corollary (McQuillan). - If $X$ is a surface of general type with $c_{1}^{2}>c_{2}$, then all entire curves of $X$ are algebraically degenerate.

It turns out that McQuillan's proof is rather involved and goes far beyond the methods presented here (see also M. Brunella [Bru98] for an enlightening 
presentation of McQuillan's main ideas). Since we do not need the full force of McQuillan's results, we present here special cases of our 1-jet and 2-jet techniques, which should in principle be quite sufficient to deal with our application (modulo a formal computational check which will not be handled here).

6.3. Proposition. - Let $X$ be a minimal surface of general type, equipped with an algebraic multi-foliation $\mathcal{F} \subset S^{m} T_{X}^{\star}$. Assume that

$$
m\left(c_{1}^{2}-c_{2}\right)+c_{1} \cdot c_{1}(\mathcal{F})>0 .
$$

Then there is a curve $\Gamma$ in $X$ such that all parabolic leaves of $\mathcal{F}$ are contained in $\Gamma$.

Proof. - Notice that every rank 1 torsion free sheaf on a surface is locally free. The inclusion morphism of $\mathcal{F}$ in $S^{m} T_{X}^{\star}$, viewed as a section of $S^{m} T_{X}^{\star} \otimes \mathcal{F}^{-1}$, defines a section of $\mathcal{O}_{X_{1}}(m) \otimes \pi^{\star} \mathcal{F}^{-1}$ whose zero divisor $Z \subset X_{1}=P\left(T_{X}\right)$ is precisely the divisor associated with the foliation (as explained in the introduction). Therefore $Z=m u-\pi^{\star} \mathcal{F}$ in $\operatorname{Pic}\left(X_{1}\right)$, and our calculations of section 2 (see $(\dagger)$ and $(\dagger \dagger)$ ) imply that $\mathcal{O}_{X_{1}}(1)_{\mid Z}$ is big as soon as

$$
\left(u_{\mid Z}\right)^{2}=m\left(c_{1}^{2}-c_{2}\right)+c_{1} \cdot \mathcal{F}>0, \quad\left(u_{\mid Z}\right) \cdot\left(-c_{1}\right)=m c_{1}^{2}+c_{1} \cdot \mathcal{F}>0 .
$$

However, as $X$ is minimal, we have $c_{2} \geq 0$, and Proposition 6.3 follows.

Again, the above 1-jet result is not sufficient to cover the case of surfaces in $\mathbb{P}^{3}$, so we have to deal with a 2-jet version instead. Let $Z \subset X_{1}=P\left(T_{X}\right)$ be the divisor associated with the given foliation $\mathcal{F}$, and $\sigma \in H^{0}\left(X_{1}, \mathcal{O}_{X_{1}}(m) \otimes \pi^{\star} \mathcal{F}^{-1}\right)$ the corresponding section. We let $\mathcal{T}_{Z}$ be the tangent sheaf to $Z$, i.e. the rank 2 sheaf $\mathcal{T}_{Z}$ defined by the exact sequence

$$
0 \longrightarrow \mathcal{T}_{Z} \longrightarrow T_{X_{1} \mid Z} \stackrel{d \sigma}{\longrightarrow} \mathcal{O}_{X_{1}}(m)_{\mid Z} \otimes \pi^{\star} \mathcal{F}_{\mid Z}^{-1} \longrightarrow 0 .
$$

If we define $\mathcal{S}=\mathcal{T}_{Z} \cap \mathcal{O}\left(V_{1}\right)$ sheaf-theoretically, we find an exact sequence

$$
0 \longrightarrow \mathcal{S} \longrightarrow V_{1 \mid Z} \stackrel{d \sigma}{\longrightarrow} \mathcal{O}_{X_{1}}(m)_{\mid Z} \otimes \pi^{\star} \mathcal{F}_{\mid Z}^{-1},
$$

where $\mathcal{S}$ is an invertible subsheaf, and a dual exact sequence

$$
0 \longrightarrow \mathcal{O}_{X_{1}}(-m)_{\mid Z} \otimes \pi^{\star} \mathcal{F}_{\mid Z} \longrightarrow V_{1 \mid Z}^{\star} \longrightarrow \mathcal{S}^{\star}
$$

We can then lift $Z$ into a surface $\widetilde{Z} \subset X_{2}$, in such a way that the projection map $\pi_{2,1}: \widetilde{Z} \rightarrow Z$ is a modification; at a generic point $x \in Z$, the point of $\widetilde{Z}$ lying above $x$ is taken to be $\left(x,\left[\mathcal{S}_{x}\right]\right) \in X_{2}$. Our goal is to compute the cohomology class of the 2-cycle $\widetilde{Z}$ in $H^{\bullet}\left(X_{2}\right)$. One of the difficulties is that the cokernel of the map

$$
d \sigma_{\mid V_{1 \mid Z}}: V_{1 \mid Z} \rightarrow \mathcal{O}_{X_{1}}(m)_{\mid Z} \otimes \pi^{\star} \mathcal{F}_{\mid Z}^{-1}
$$

may have torsion along a 1 -cycle $G_{1} \subset Z$, i.e., there is a factorization

$$
d \sigma_{\mid V_{1 \mid Z}}: V_{1 \mid Z} \rightarrow \mathcal{O}_{X_{1}}(m)_{\mid Z} \otimes \pi^{\star} \mathcal{F}_{\mid Z}^{-1} \otimes \mathcal{O}_{Z}\left(-G_{1}\right) \rightarrow \mathcal{O}_{X_{1}}(m)_{\mid Z} \otimes \pi^{\star} \mathcal{F}_{\mid Z}^{-1}
$$

such that the cokernel of the first arrow has 0-dimensional support (of course, $G_{1}$ need not be reduced). If the foliation is generic, however, the cokernel of $d \sigma_{\mid V_{1 \mid Z}}$ will have no torsion in codimension 1 , and $d \sigma$ then induces a section of

$$
\mathcal{O}_{X_{2}}(1) \otimes \pi_{2,1}^{\star} \mathcal{O}_{X_{1}}(m) \otimes \pi_{2,0}^{\star} \mathcal{F}_{\mid \pi_{2,1}^{-1}(Z)}^{-1} \sim\left(u_{2}+m u_{1}-\mathcal{F}\right)_{\mid \pi_{2,1}^{-1}(Z)}
$$


whose zero locus is $\widetilde{Z}$. As $Z \sim m u_{1}-\mathcal{F}$, the cohomology class of $\widetilde{Z}$ in $H^{4}\left(X_{2}\right)$ is given by

$$
\begin{aligned}
\{\widetilde{Z}\} & =\left(m u_{1}-\mathcal{F}\right) \cdot\left(u_{2}+m u_{1}-\mathcal{F}\right) \\
& =m^{2} u_{1}^{2}+m u_{1} \cdot u_{2}-2 m u_{1} \cdot \mathcal{F}-u_{2} \cdot \mathcal{F}+\mathcal{F}^{2} .
\end{aligned}
$$

A short Chern class computation yields

$$
\left(2 u_{1}+u_{2}\right)^{2} \cdot \widetilde{Z}=m^{2}\left(4 c_{1}^{2}-3 c_{2}\right)+m\left(5 c_{1}^{2}-3 c_{2}\right)+(8 m+4) c_{1} \cdot \mathcal{F}+3 \mathcal{F}^{2} .
$$

If the 1-cycle $G_{1}$ is nonzero, our numerical formula for $\widetilde{Z}$ becomes

$$
\{\widetilde{Z}\}=\left(m u_{1}-\mathcal{F}\right) \cdot\left(u_{2}+m u_{1}-\mathcal{F}\right)-\pi_{2,1}^{\star}\left\{G_{1}\right\} .
$$

On the other hand, we find

$$
\left(2 u_{1}+u_{2}\right)^{2} \cdot \pi_{2,1}^{\star}\left\{G_{1}\right\}=\left(3 u_{1}-c_{1}\right) \cdot G_{1} .
$$

The general formula for $\left(2 u_{1}+u_{2}\right)^{2} \cdot \widetilde{Z}$ is thus

$\left(2 u_{1}+u_{2}\right)^{2} \cdot \widetilde{Z}=m^{2}\left(4 c_{1}^{2}-3 c_{2}\right)+m\left(5 c_{1}^{2}-3 c_{2}\right)+(8 m+4) c_{1} \cdot \mathcal{F}+3 \mathcal{F}^{2}-\left(3 u_{1}-c_{1}\right) \cdot G_{1}$.

By using obvious exact sequences, $H^{2}\left(\widetilde{Z}, m \mathcal{O}_{X_{2}}(2,1) \widetilde{Z}_{\mid \widetilde{Z}}\right)$ is a quotient of

$$
H^{2}\left(\pi_{2,1}^{-1}(Z), m \mathcal{O}_{X_{2}}(2,1)_{\mid \pi_{2,1}^{-1}(Z)}\right)
$$

which is itself controlled by $H^{2}\left(X_{2}, m \mathcal{O}_{X_{2}}(2,1)\right), H^{3}\left(X_{2}, m \mathcal{O}_{X_{2}}(2,1) \otimes \mathcal{O}(-Z)\right)$. A direct image argument shows that the latter groups are controlled by groups of the form $H^{2}\left(X, E_{2,3 m} T_{X}^{\star} \otimes L\right)$, with suitable line bundles $L$. As in the proof of Theorem 3.4 (possibly after changing $\mathcal{O}_{X_{2}}(2,1)$ into $\mathcal{O}_{X_{2}}(2+\varepsilon, 1)$ in the above arguments), one can check that the latter $H^{2}$ groups vanish. The positivity of $\left(2 u_{1}+u_{2}\right)^{2} \cdot \widetilde{Z}$ thus implies that $\mathcal{O}_{X_{2}}(2,1)_{\mid \widetilde{Z}}$ is big, and therefore all parabolic leaves of the (multi)-foliation $\mathcal{F}$ are algebraically degenerate. We thus obtain:

6.4. Proposition. - Let $X$ be a surface of general type, equipped with a multifoliation $\mathcal{F} \subset S^{m} T_{X}^{\star}$, and let $\sigma \in H^{0}\left(X_{1}, \mathcal{O}_{X_{1}}(m) \otimes \pi_{1,0}^{\star} \mathcal{F}^{\star}\right)$ be the associated canonical section. Finally, let $G_{1}$ be the divisorial part of the subscheme defined by coker $\left(d \sigma_{\mid V_{1 \mid Z}}\right)$. Then, under the assumption

$$
m^{2}\left(4 c_{1}^{2}-3 c_{2}\right)+m\left(5 c_{1}^{2}-3 c_{2}\right)+(8 m+4) c_{1} \cdot \mathcal{F}+3 \mathcal{F}^{2}-\left(3 u_{1}-c_{1}\right) \cdot G_{1}>0,
$$

all parabolic leaves of $\mathcal{F}$ are algebraically degenerate.

6.5. Corollary. - Let $X \subset \mathbb{P}^{3}$ be a surface of degree $d \geq 18$ with $\operatorname{Pic}(X)=\mathbb{Z}$, and let $\mathcal{F} \subset S^{m} T_{X}^{\star}$ be a generic multi-foliation, in the sense that the 1-cycle $G_{1}$ defined above is zero. Then all parabolic leaves of $\mathcal{F}$ are algebraically degenerate and contained in a fixed 1-dimensional algebraic subset $Y \subset X$.

Proof. - Note that the line subbundle $\mathcal{F} \subset S^{m} T_{X}^{\star}$ must be negative (otherwise $\mathcal{F}$ would yield a nontrivial section of $\left.S^{m} T_{X}^{\star}\right)$, hence $c_{1} \cdot \mathcal{F}>0, \mathcal{F}^{2}>0$, and likewise we have

$$
4 c_{1}^{2}-3 c_{2}=d\left(d^{2}-20 d+46\right)>0, \quad 5 c_{1}^{2}-3 c_{2}=d\left(2 d^{2}-28 d+62\right)>0
$$

for $d \geq 18$. Thus, we get the conclusion if $G_{1}=0$ (but a rather large additional contribution of $G_{1}$ would still be allowable; we do not know how much of it can actually occur). 


\section{Proof of the Corollaries}

\section{Proof of Corollary 1.}

Recall that by the Noether-Lefschetz theorem, a very generic surface $X$ in $\mathbb{P}^{3}$ is such that $\operatorname{Pic}(X)=\mathbb{Z}$, with generator $\mathcal{O}_{X}(1)$. On the other hand, improving a result of $\mathrm{H}$. Clemens ([Cl86] and [CKM88]), G. Xu [Xu94] has shown that the genus of every curve contained in a very generic surface of degree $d \geq 5$ satisfies the bound $g \geq d(d-3) / 2-2$ (this bound is sharp). In particular, such a surface does not contain rational or elliptic curves. Now take a very generic surface in $\mathbb{P}^{3}$ of degree $d \geq 21$, which has no rational or elliptic curves, and such that the conclusions of the Main Theorem apply, i.e. every nonconstant entire curve $f: \mathbb{C} \rightarrow X$ is such that $f_{[1]}(\mathbb{C})$ lies in the leaf of an algebraic foliation on a surface $Z \subset X_{1}$. Then, by McQuillan's result, $f$ must be algebraically degenerate. The closure $\Gamma=\overline{f(\mathbb{C})}$ would then be an algebraic curve of genus 0 or 1 , contradiction.

7.1. Remark. - If one would like to avoid any appeal to McQuillan's deep result, it would remain to check on an example that the multi-foliation defined by $Z$ satisfies the sufficient condition described in Proposition 6.4. This might require for instance a computer check, and is likely to hold without much restriction.

7.2. Remark. - It is extremely likely that Corollary 1 holds true for generic surfaces and not only for very generic ones. In fact, since we have a smooth family of nonsingular surfaces $X \rightarrow M_{d} \subset \mathbb{P}^{N_{d}}$ in each degree $d$, the Riemann-Roch calculations of sections 3,4 hold true in the relative situation, and thus produce an algebraic family of divisors $Z_{t} \subset\left(X_{t}\right)_{2}$ on some Zariski open subset $M_{d}^{\prime} \subset M$, $t \in M_{d}^{\prime}$. By shrinking $M_{d}^{\prime}$, we can assume that all $z_{t}$ are irreducible, and that we have a flat family $z \rightarrow M_{d}^{\prime}$. By relative Riemann-Roch again, we get a family of divisors $y_{t} \subset z_{t}$, and thus a family of foliations $\mathcal{F}_{t}$ on the 1-jet bundles $\left(X_{t}\right)_{1}$. Finally, if Proposition 6.4 can be applied to these foliations (and we strongly expect that this is indeed the case), we get an algebraic family of curves $\Gamma_{t} \subset X_{t}$ such that all holomorphic maps $f: \mathbb{C} \rightarrow X_{t}$ are contained in $\Gamma_{t}$. As the degree is bounded, a trivial Hilbert scheme argument implies that the set of $t$ 's for which one of the components of $\Gamma_{t}$ is rational or elliptic is closed algebraic and nowhere dense. Our claim follows.

\section{Proof of Corollary 2.}

Let $C=\sigma^{-1}(0)$ be a nonsingular curve of degree $d$ in $\mathbb{P}^{2}$. Consider the cyclic covering $X_{C}=\left\{z_{3}^{d}=\sigma\left(z_{0}, z_{1}, z_{2}\right)\right\} \rightarrow \mathbb{P}^{2}$ of degree $d$, ramified along $C$. Then $X_{C}$ is a nonsingular surface in $\mathbb{P}^{3}$, and as $\mathbb{C}$ is simply connected, every holomorphic map $f: \mathbb{C} \rightarrow \mathbb{P}^{2} \backslash C$ can be lifted to $X_{C}$. It is known that $\operatorname{Pic}\left(X_{C}\right)=\mathbb{Z}$ for generic $C$; see e.g. J. Esser's PhD Thesis [Ess93] (we express our thanks to K. Amerik and E. Viehweg for pointing out the reference to us; see Hartshorne [Ha75] for the following related well known fact: if $\left(X_{t}\right)_{t \in \mathbb{P}^{1}}$ is a Lefschetz pencil of surfaces on a 3 -fold $W$ and $H^{0}\left(X_{t}, K_{X_{t}}\right) \neq 0$, then $\operatorname{Pic}\left(X_{t}\right) \simeq \operatorname{Pic}(W)$ for generic $\left.t\right)$. The 
nonexistence theorem proved in section 6 also holds true for at least one $X_{C}$, for example

$$
\begin{aligned}
C & =\left\{z_{0}^{d}+z_{1}^{d}+z_{2}^{d}+a z_{0}^{k_{0}} z_{1}^{k_{1}} z_{2}^{k_{2}}=0\right\}, \\
X_{C} & =\left\{z_{0}^{d}+z_{1}^{d}+z_{2}^{d}+z_{3}^{d}+a z_{0}^{k_{0}} z_{1}^{k_{1}} z_{2}^{k_{2}}=0\right\} .
\end{aligned}
$$

We then conclude as above that $X_{C}$ is hyperbolic for generic $C$. This implies in particular that $\mathbb{P}^{2} \backslash C$ is hyperbolic and hyperbolically embedded in $\mathbb{P}^{2}$ (see Green [Gr77]).

\section{References}

[BPV84] Barth W., Peters C., Van de Ven A. - Compact complex surfaces, Ergebrnisse der Mathematik und ihrer Grenzgebiete, 3, Folge, Band 4, Springer, 1984.

[Bo77] Bogomolov F.A. - Families of curves on a surface of general type, Soviet. Math. Dokl., 18 (1977), 1294-1297.

[Bo79] Bogomolov F.A. - Holomorphic tensors and vector bundles on projective varieties, Math. USSR Izvestija, 13 (1979), 499-555.

[Bro78] Brody, R. - Compact manifolds and hyperbolicity, Trans. Amer. Math. Soc., 235 (1978), 213-219.

[Bru98] Brunella M. - Courbes entières et feuilletages holomorphes, L'Enseignement Mathématique, 45 (1999), 195-216.

[CKM88] Clemens H., Kollár J., Mori S. - Higher dimensional complex geometry, Astérisque 166, 1988.

[Cl86] Clemens H. - Curves on generic hypersurface, Ann. Sci. Éc. Norm. Sup., 19 (1986), 629-636.

[Dem95] Demailly J.-P. - Algebraic criteria for Kobayashi hyperbolic projective varieties and jet differentials, Proceedings of the AMS Summer Institute on Alg. Geom. held at Santa Cruz, ed. J. Kollár, July 1995, to appear.

[DEG96] Demailly J.-P., El Goul J. - Connexions méromorphes projectives et variétés algébriques hyperboliques, C. R. Acad. Sci. Paris, à paraître, (1997).

[DSW92] Dethloff, G., Schumacher, G., Wong, P.M. - Hyperbolicity of the complement of plane algebraic curves, Amer. J. Math, 117 (1995), 573-599.

[DSW94] Dethloff, G., Schumacher, G., Wong, P.M. - On the hyperbolicity of the complements of curves in Algebraic surfaces: the three component case, Duke Math. Math., 78 (1995), 193-212.

[DZ95a] Dethloff, G., Zaidenberg, M. - Plane curves with C-hyperbolic complements, Prépublication de l'Institut Fourier, 299, Grenoble 1995, 44 p, Duke Math. E-print alg-geom/9501007 (à paraître dans Annali Sc. Norm. Super. Pisa).

[DZ95b] Dethloff, G., Zaidenberg, M. - Examples of plane curves of low degrees with hyperbolic and C-hyperbolic complements, Proc. Conf. Geometric Complex Analysis, Hayama, 20-29 March, 1995, J. Noguchi, H. Fujimoto, J. Kajiwara, T. Ohsawa eds., World Scientific, Singapore, 17 p (à paraître).

[Ein88] Ein, L. - Subvarieties of generic complete intersections, Invent. Math., 94 (1988), 163-169.

[EG96] El Goul J. - Algebraic families of smooth hyperbolic surfaces of low degree in $\mathbb{P}_{\mathbb{C}}^{3}$, Manuscripta Math., 90 (1996), 521-532. 
[Ess93] Esser J. - Noether-Lefschetz-Theoreme fur zyklische belagerungen, PhD Thesis in Mathematik und Informatik der Universität Gesamthochschule Essen, February 1993.

[Gr77] Green M. - The hyperbolicity of the complement of $2 n+1$ hyperplanes in general position in $\mathbf{P}_{\mathbf{C}}^{n}$ and related results, Proc. Amer. Math. Soc., 66 (1977), 109-113.

[GG80] Green M., Griffiths P. — Two applications of algebraic geometry to entire holomorphic mappings, The Chen Symposium 1979, Proc. Inter. Sympos. Berkeley, CA, 1979, Springer-Verlag, , New York (1980), 41-74.

[Ha75] Hartshorne R. - Equivalence relations on algebraic cycles and subvarieties of small codimensions, Proc. of Symp. in Pure Math., 29 (1975), 129-164.

[Hi66] Hirzebruch F. - Topological methods in Algebraic Geometry, Grundl. Math. Wiss.131, Spriger, Heidelberg, 1966.

[Jo78] Jouanolou J.-P. - Hypersurfaces solutions d'une équation de Pfaff analytique, Math. Ann., 332 (1978), 239-248.

[Ko70] Kobayashi S. - Hyperbolic manifolds and holomorphic mappings, Marcel Dekker, New York, 1970.

[La86] Lang S. - Hyperbolic and diophantine analysis, Bull. Amer. Math. Soc., 14 (1986), 159-205.

[La87] Lang S. - Introduction to complex hyperbolic spaces, Springer-Verlag, New York, 1987.

[LY90] Lu S.S.-Y., Yau S.T. - Holomorphic curves in surfaces of general type, Proc. Nat. Acad. Sci. USA, 87 (1990), 80-82.

[M-De78] Martin-Deschamps M. - Courbes de genre géométrique borné sur une surface de type général (d'après F. Bogomolov), Séminaire Bourbaki, 519 (1978), 1-15.

[McQ97] McQuillan M. — Diophantine approximations and foliations, Preprint (1997), to appear in Publ. Math. IHES.

[Mi82] Miyaoka Y. - Algebraic surfaces with positive indices, Katata Symp. Proc. 1982, Progress in Math., 39 (1983), 281-301.

[Pa99] Paun, M. - Personal communication, January 1999.

[Sa78] Sakai F. - Symmetric powers of the cotangent bundle and classification of algebraic varieties, Proc. Copenhagen Meeting in Alg. Geom., 1978.

[ST88] Schneider M., Tancredi A. - Almost-positive vector bundles on projective surfaces, Math. Ann., 280 (1988), 537-547.

[Se68] Seidenberg A. - Reduction of singularities of the differential equation AdY= $B d X$, Amer. J. of Math., 90 (1968), 248-269.

[Siu87] Siu Y.-T. - Defect relations for holomorphic maps between spaces of different dimensions, Duke Math. J., 55 (1987), 213-251.

[SY95] Siu Y.-T., Yeung S.K. - Hyperbolicity of the complement of a generic smooth curve of high degree in the complex projective plane, to appear in Inventiones Math., 1996.

[SY97] Siu Y.-T., Yeung S.K. — Defects for ample divisors of abelian varieties, Schwarz lemma and hyperbolic hypersurfaces of low degrees, Preprint, Fall 1996.

[Voi96] Voisin, C. - On a conjecture of Clemens on rational curves on hypersurfaces, J. Differ. Geom, 44 (1996), 200-213.

[Xu94] Xu G. - Subvarieties of general hypersurfaces in projective space, J. Differential Geometry, 39 (1994), 139-172.

[Yau78] Yau S.T. - On the Ricci curvature of a compact Kähler manifold and the complex Monge Ampère equation, Comm. Pure and Appl. Math., 31 (1978), 339-441.

[Zai87] Zaidenberg, M. - The complement of a generic hypersurface of degree $2 n$ in $\mathbb{C P}^{n}$ is not hyperbolic, Siberian Math. J., 28 (1987), 425-432. 
[Zai89] Zaidenberg, M. - Stability of hyperbolic embeddedness and construction of examples, Math. USSR Sbornik, 63 (1989), 351-361.

[Zai93] Zaidenberg, M. - Hyperbolicity in projective spaces, International Symposium on Holomorphic mappings, Diophantine Geometry and Related topics, R.I.M.S. Lecture Notes ser. 819, R.I.M.S. Kyoto University (1993), 136-156.

(printed on March 19, 2018, 17:28)

Université Joseph Fourier Grenoble I

Institut Fourier (Mathématiques)

UMR 5582 du C.N.R.S., BP 74

38402 Saint-Martin d'Hères, France

e-mail: demailly@fourier.ujf-grenoble.fr, elgoul@picard.ups-tlse.fr 